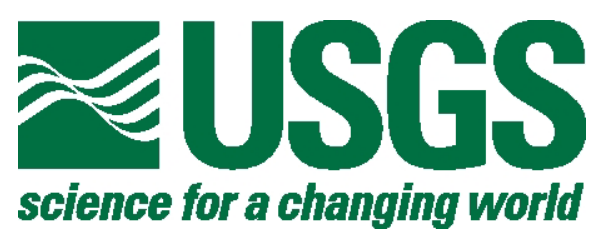

\title{
Stratigraphic inferences derived from borehole data of Tertiary basin-filling rocks of the Pahrump Valley basin, Nevada and California
}

By Donald S. Sweetkind ${ }^{1}$, Emily Taylor ${ }^{1}$, and Heather Putnam ${ }^{2}$

This report is preliminary and has not been reviewed for conformity with U.S. Geological Survey editorial standards or with the North American Stratigraphic Code.

Any use of trade, product, or firm names is for descriptive purposes only and does not imply endorsement by the U.S. Government.

Open-File Report 2003-03-051

${ }^{1}$ Denver, $\mathrm{CO}$

${ }^{2}$ Las Vegas, NV

U.S. Department of the Interior

U.S. Geological Survey 


\section{Contents}

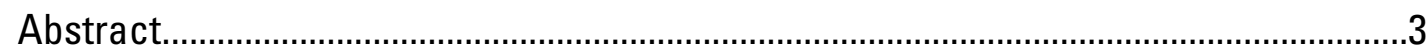

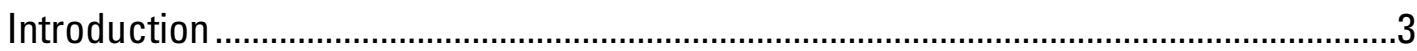

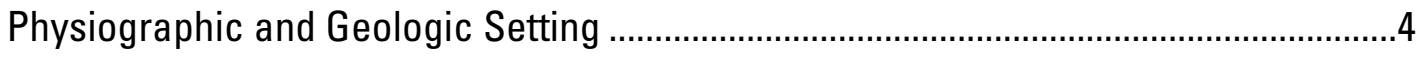

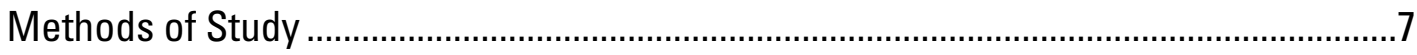

Borehole Data...........................................................................................................

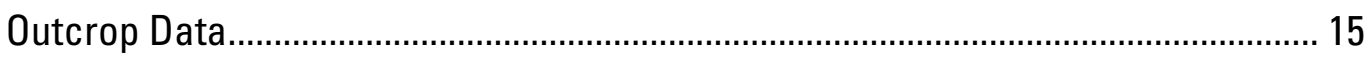

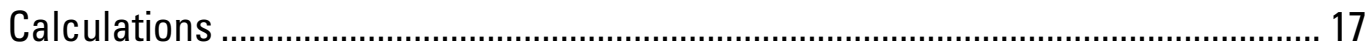

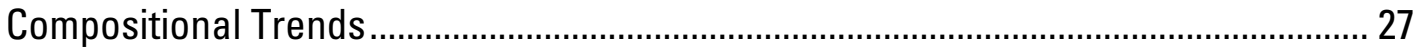

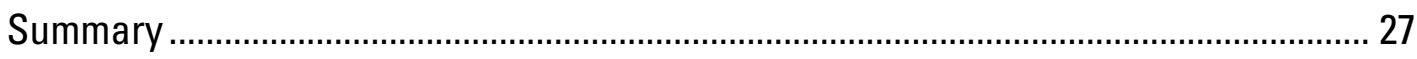

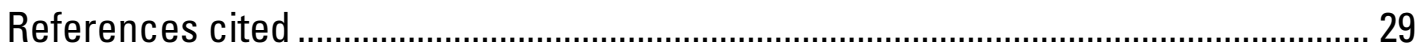

\section{Figures}

1. Location and general features of the Pahrump Valley .............................................

2. Location map of boreholes and outcrops in Pahrump Valley used in this

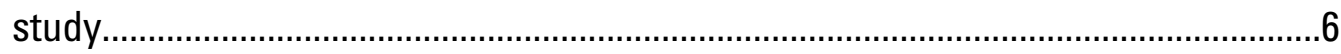

3. Example stratigraphic sections from the Pahrump Valley ...................................... 16

4. Sample borehole showing methodology for calculation of coarse grained sedimentary component........................................................................... 18

5. Contoured lithologic data from Pahrump Valley .................................................... 28

\section{Tables}

1. Location and depth of Pahrump Valley boreholes ............................................. 8

2. Lithologic descriptors derived from borehole logs ........................................... 15

3. Lithologic descriptors derived from outcrops in Wheeler Wash ......................... 17

4. Lithologic data from Pahrump Valley boreholes .................................................. 19 


\title{
Stratigraphic analysis from borehole data of Tertiary basin-filling rocks of the Pahrump Valley basin, Nevada and California
}

\author{
By Donald S. Sweetkind, Emily Taylor and Heather Putnam
}

\begin{abstract}
This report summarizes the results from the compilation and interpretation of lithologic information from 266 driller's logs from the Pahrump Valley, NV-CA. Borehole records for the Pahrump Valley were selected from a total of several thousand available well logs as those having lithologic data and high confidence in the well locations. Driller's descriptions were reduced into one of 14 lithologic categories to produce a set of subsurface data that were internally consistent and amenable to contouring. Using the lithologic descriptors, the aggregate thickness of general lithologic types (coarse grained material, fine grained material, limestone, and volcanic material) were computed for each borehole. These data were used to compute ratios such as percent coarse-grained component of sedimentary rocks penetrated in each borehole that could be contoured.
\end{abstract}

\section{Introduction}

Management decisions regarding limited ground-water resources in the desert southwestern U.S. require knowledge of the stratigraphy and material properties of Cenozoic basin-filling rocks that are often covered with young alluvial material. Of interest is the development of methodologies for the rapid compilation and interpretation of readily available subsurface information. This report assembles the available drill-hole lithologic data for the basin-filling rocks in the Pahrump Valley, NV-CA, augmented with limited field data. Compilation and interpretation of these data represent the initial step in developing a three-dimensional understanding of the geometry and properties of the subsurface materials within this basin.

Agricultural and residential development in the Pahrump Valley has resulted in the drilling of many water wells from which lithologic information is available. This report summarizes the results from the compilation and interpretation of lithologic information from 266 driller's logs from the Pahrump Valley, NV-CA. These data were augmented by outcrop observations of alluvial fan material in Wheeler Wash, an incised drainage on the east side of the valley. This area was chosen for its relatively close spacing of boreholes and because the location of many of these boreholes at the transition from alluvial fan to basin-axis made it likely that distinct lithologic successions from each depositional environment could be recognized. This report develops a methodology for characterizing lithologic changes and variations in subsurface material properties based on analysis of lithologic information from driller's logs from wells in the Pahrump Valley. 


\section{Physiographic and Geologic Setting}

The Pahrump Valley is an alluvial basin along the Nevada-California border that covers about 2700 square kilometers in Nye and Clark Counties, NV, and Inyo and San Bernardino Counties, CA (fig. 1). The Spring Mountains are the dominant topographic feature on the northeastern and eastern borders of the basin. The topographically lower Montgomermy Mountains, the Nopah Range, and the Kingston Range bound the basin to the northwest, west, and southwest, respectively. The Pahrump Valley is a closed basin with no surface outflows.

All of the ranges that surround the Pahrump Valley are composed of Paleozoic and Late Proterozoic carbonate and clastic rocks (Longwell and others, 1965; Burchfiel and others, 1974; Burchfiel and others, 1982; 1983). In addition, the Kingston Range contains a large Tertiary granitic pluton (Calzia and others, 2000). Surficial geologic maps show that the uppermost basin-fill consists of unconsolidated coarse-grained alluvial materials that have been deposited near the sides of the valley and fine-grained basin axis, playa, and spring and associated marsh or wetland deposits that occupy the central parts of the valley (Malmberg, 1967; Lundstrom and others, in press; Page and others, in press). The northeastern side of the basin is characterized by large alluvial fans, the most prominent of which are the coalesced Pahrump and the Manse Fans (fig. 1) (Malmberg, 1967; Harrill, 1986).

The right-lateral Pahrump-Stewart Valley Fault Zone (Stewart and others, 1968; Stewart and Crowell, 1992) roughly parallels the California-Nevada boundary through Stewart Valley and Pahrump Valley (PSV, fig. 2). Quaternary deposits almost everywhere bury the fault zone (Hoffard, 1991; Workman and others, 2002). Gravity data show that the fault is associated with complex variations in the elevation of the Paleozoic-Tertiary contact (Blakely and others, 1998; Blakely and others, 1999). The fault zone forms a buried transpressional bedrock ridge separating two steep-sided, probably fault-bounded basins (Wright, 1989; Blakely and others, 1998). The well data described in this report all come from the part of the Pahrump basin that lies to the northeast of the fault zone. The relatively shallow wells described here test only the upper parts of what may be kilometers-deep Tertiary basins adjacent to the fault zone (Blakely and others, 1998; Blakely and others, 1999).

The other structural features of interest for this part of the Pahrump basin are the range-front faults on the western side of the Spring Mountains. These faults include the West Spring Mountains Fault (WSM, fig. 2) (Hoffard, 1991; Piety, 1994; Anderson and others, 1995) and the Grapevine Fault (GV, fig. 2) (Burchfiel and others, 1983). These faults have predominantly normal, dip-slip, west-side-down offset; the West Spring Mountains Fault has documented Quaternary offset (Hoffard, 1991; Piety, 1994; Anderson and others, 1995). These faults lie mostly to the east of the wells discussed in this report. A single well at the northern end of Pahrump Valley lies on the east side of the West Spring Mountains Fault; all of the four outcrop stations are located east of the West Spring Mountains Fault. 


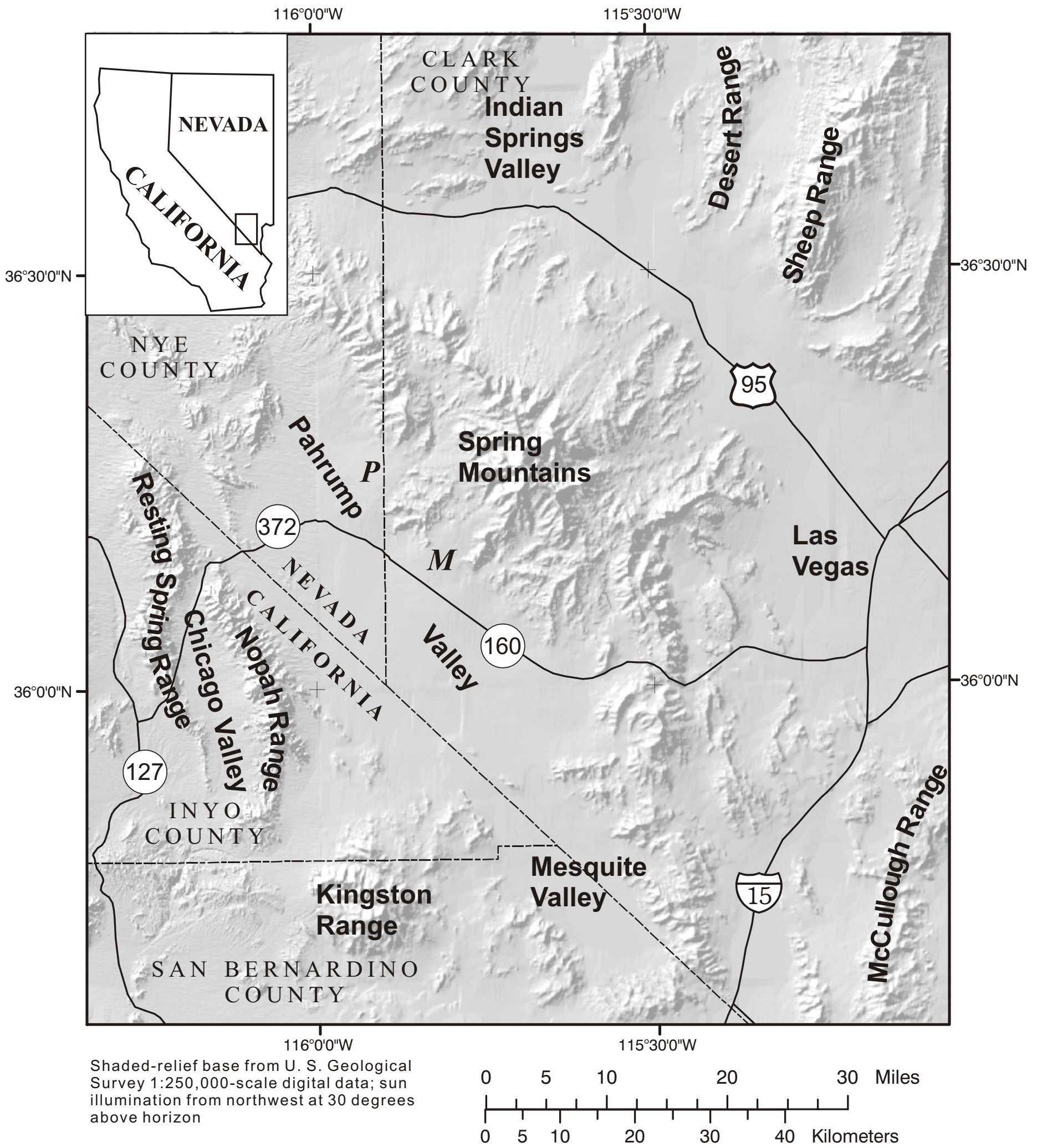

Figure 1. Location map of the Pahrump Valley. Location of the Pahrump and Manse fans are denoted by $\mathrm{P}$ and $\mathrm{M}$, respectively. 


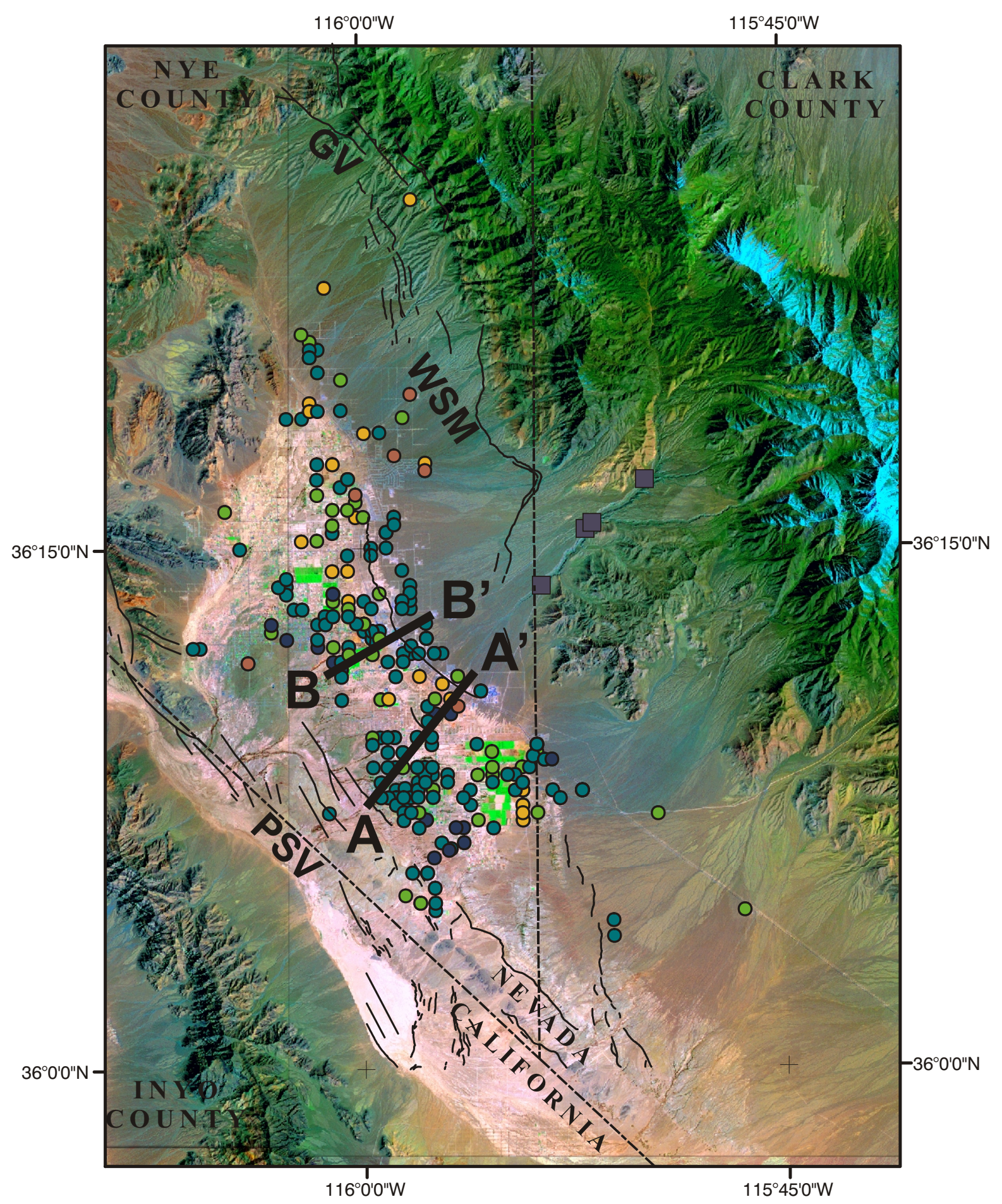

False-color composite LANDSAT 6

thematic mapper image displays spectral bands 2, 5 and 7 in RGB space. Bedrock exposures of Paleozoic carbonate rocks appear in shades of blue and green;

Proterozoic clastic rocks appear in redbrown tones. Playa and basin-axis deposits appear in white or light pink

tones. Irrigated domestic and agricultural

land appears as bright green.

Figure 2. Location map of boreholes (circles) and outcrop stations (blue squares) in Pahrump Valley used in this study. Stratigraphic sections A-A' and B-B' are shown in figure 3. Locations of West Spring Mountains fault (WSM), Grapevine fault (GV), and Pahrump-Stewart Valley fault (PSV) after Anderson and others (1995) and Workman and others (2002).

Thickness of
drilled interval, m
○ $12-75$
o $76-150$
o $151-225$
o $226-300$
o $301-375$




\section{Methods of Study}

\section{Borehole data}

More than 700 borehole records for the Pahrump basin were acquired from the State of Nevada Engineer's Office. These records were selected from a total of several thousand well logs as those having lithologic data and high confidence in the well locations. These records were further reduced to a data set of 266 boreholes, using the procedures described below, to produce a set of subsurface data that were internally consistent and amenable to contouring.

Paper borehole records were transcribed into digital files. Data that were transcribed from the driller's logs include well name, owner, location, total depth, water table elevation, and a series of downhole intervals for which the elevation of the top and bottom of the interval and the lithologic description for each interval was recorded. Borehole locations as reported in Section, Township, Range format were converted to latitude and longitude and easting and northing (in UTM meters, using UTM zone 11, NAD 27 coordinates). Borehole collar elevations were assigned by intersecting the collar location with a grid based on a USGS 7.5' digital elevation model of the area.

From the initial set of more than 700 logs for the Pahrump basin, several criteria were used to reduce the number of holes. Boreholes were chosen to provide a broad spatial distribution, include the deepest boreholes, include holes with the most continuous data, and include those holes that were representative of a closely spaced group of boreholes. Wells drilled into the basin-fill aquifer range from several tens of meters to more than 300 meters deep (fig. 2). With the exception of one or two wells near the margin of the basin, the wells do not fully penetrate the basin fill. Most boreholes less than $90 \mathrm{~m}$ were discarded; care was taken to ensure that this did not result in data gaps. Holes were discarded where the borehole location was uncertain, for example, where a hole could not be located to greater precision than a full section. Holes were discarded where lithologic information was unavailable or where there were large intervals that lacked lithologic descriptions. Table 1 contains location data for the resulting boreholes; figure 2 shows their location in Pahrump Valley. 
Table 1. Location and depth of Pahrump Valley boreholes

[Data are compiled from the State of Nevada, Division of Water Resources, Well Driller's Reports;

Easting and northing are in UTM meters, using UTM zone 11, NAD 27 coordinates; corrected thickness is computed by subtracting from the total depth of the hole any intervals that lacked lithologic descriptions and intervals described as soil]

\begin{tabular}{|c|c|c|c|c|c|}
\hline Index & Well name & $\begin{array}{c}\text { Easting } \\
\text { (UTM m) }\end{array}$ & $\begin{array}{l}\text { Northing } \\
\text { (UTM m) }\end{array}$ & $\begin{array}{l}\text { Total depth } \\
(\mathrm{m})\end{array}$ & $\begin{array}{l}\text { Corrected } \\
\text { depth }(\mathrm{m})\end{array}$ \\
\hline 1 & PB10033 & 594469 & 3999910 & 91.4 & 89.3 \\
\hline 2 & PB10059 & 592137 & 3998282 & 111.3 & 111.3 \\
\hline 3 & PB10088 & 592082 & 4001086 & 93.6 & 93.6 \\
\hline 4 & PB1013 & 589546 & 4014402 & 199.6 & 199.6 \\
\hline 5 & PB10142 & 582589 & 4013871 & 155.4 & 154.5 \\
\hline 6 & PB1024 & 590016 & 4007535 & 93.0 & 90.6 \\
\hline 7 & PB 10274 & 590000 & 4009138 & 125.0 & 125.0 \\
\hline 8 & PB10369 & 581285 & 4006587 & 167.9 & 146.7 \\
\hline 9 & PB10371 & 591649 & 4001883 & 129.8 & 128.9 \\
\hline 10 & PB10402 & 591649 & 4001883 & 110.9 & 109.1 \\
\hline 11 & PB10403 & 590874 & 4001874 & 121.9 & 121.3 \\
\hline 12 & PB10456 & 593847 & 3992661 & 92.0 & 90.8 \\
\hline 13 & PB10641 & 596026 & 4001529 & 137.8 & 130.2 \\
\hline 14 & PB10706 & 586284 & 4008669 & 99.1 & 97.6 \\
\hline 15 & PB 10770 & 592899 & 3999492 & 117.3 & 117.3 \\
\hline 16 & PB10792 & 587540 & 4007880 & 121.9 & 120.7 \\
\hline 17 & PB10916 & 591728 & 3999079 & 124.7 & 122.9 \\
\hline 18 & PB10918 & 592202 & 3999177 & 106.4 & 105.8 \\
\hline 19 & PB10920 & 591691 & 4000281 & 106.4 & 106.4 \\
\hline 20 & PB1093 & 599225 & 4001534 & 222.5 & 221.6 \\
\hline 21 & PB10951 & 588385 & 4005886 & 106.7 & 70.1 \\
\hline 22 & PB10952 & 587552 & 4006678 & 106.7 & 70.1 \\
\hline 23 & PB10953 & 593834 & 4003138 & 106.7 & 70.1 \\
\hline 24 & PB10954 & 594634 & 4003147 & 106.7 & 68.2 \\
\hline 25 & PB10977 & 588813 & 4005489 & 106.7 & 70.1 \\
\hline 26 & PB11018 & 599225 & 4001534 & 137.2 & 137.2 \\
\hline 27 & PB11205 & 590840 & 4007544 & 91.4 & 90.2 \\
\hline 28 & PB11301 & 595678 & 3999091 & 106.7 & 105.5 \\
\hline 29 & PB11348 & 585872 & 4009897 & 99.1 & 93.0 \\
\hline 30 & PB11478 & 599225 & 4001534 & 198.7 & 55.4 \\
\hline 31 & PB11806 & 590445 & 4007139 & 128.0 & 126.8 \\
\hline 32 & PB11973 & 588352 & 4009121 & 184.4 & 182.9 \\
\hline 33 & PB12038 & 599229 & 4001133 & 222.5 & 217.0 \\
\hline 34 & PB12163 & 593703 & 3999100 & 152.4 & 152.4 \\
\hline 35 & PB12187 & 585877 & 4009466 & 128.6 & 124.0 \\
\hline 36 & PB12494 & 592526 & 4006360 & 91.4 & 91.4 \\
\hline 37 & PB12761 & 589201 & 4006695 & 106.7 & 105.5 \\
\hline 38 & PB12762 & 591715 & 4005119 & 106.7 & 98.8 \\
\hline 39 & PB12763 & 588809 & 4005890 & 106.7 & 105.5 \\
\hline 40 & PB12765 & 588817 & 4005089 & 106.7 & 105.2 \\
\hline 41 & PB12928 & 592526 & 4006360 & 91.4 & 91.4 \\
\hline 42 & PB12996 & 593669 & 3999901 & 91.4 & 91.1 \\
\hline
\end{tabular}


Table 1. Location and depth of Pahrump Valley boreholes - Continued.

[Data are compiled from the State of Nevada, Division of Water Resources, Well Driller's Reports;

Easting and northing are in UTM meters, using UTM zone 11, NAD 27 coordinates; corrected thickness is computed by subtracting from the total depth of the hole any intervals that lacked lithologic descriptions and intervals described as soil]

\begin{tabular}{|c|c|c|c|c|c|}
\hline Index & Well name & $\begin{array}{c}\text { Easting } \\
\text { (UTM m) }\end{array}$ & $\begin{array}{l}\text { Northing } \\
\text { (UTM m) }\end{array}$ & $\begin{array}{l}\text { Total depth } \\
\text { (m) }\end{array}$ & $\begin{array}{l}\text { Corrected } \\
\text { depth }(\mathrm{m})\end{array}$ \\
\hline 43 & PB12999 & 592101 & 4006355 & 91.4 & 90.5 \\
\hline 44 & PB13014 & 587495 & 4012348 & 198.1 & 167.6 \\
\hline 45 & PB13351 & 596245 & 4004366 & 91.4 & 89.6 \\
\hline 46 & PB13425 & 593265 & 4000297 & 91.4 & 90.8 \\
\hline 47 & PB14191 & 585072 & 4007455 & 243.8 & 152.4 \\
\hline 48 & PB14429 & 593627 & 4001503 & 91.4 & 89.6 \\
\hline 49 & PB14886 & 593414 & 4002733 & 106.7 & 105.2 \\
\hline 50 & PB14887 & 593405 & 4003534 & 106.7 & 104.3 \\
\hline 51 & PB 14888 & 588348 & 4009521 & 167.6 & 149.9 \\
\hline 52 & PB15366 & 583382 & 4011876 & 91.7 & 90.8 \\
\hline 53 & PB1588 & 598439 & 4000323 & 204.2 & 204.2 \\
\hline 54 & PB16088 & 583839 & 4005810 & 91.4 & 90.5 \\
\hline 55 & PB16112 & 596869 & 3999905 & 152.4 & 152.4 \\
\hline 56 & PB16144 & 590441 & 4007540 & 96.3 & 94.8 \\
\hline 57 & PB16282 & 597668 & 3999914 & 122.5 & 121.6 \\
\hline 58 & PB16283 & 591332 & 3998675 & 91.4 & 88.4 \\
\hline 59 & PB1664 & 598478 & 3999091 & 227.1 & 216.7 \\
\hline 60 & PB1702 & 598487 & 3998290 & 242.3 & 204.2 \\
\hline 61 & PB1826 & 589134 & 4010731 & 234.4 & 234.4 \\
\hline 62 & PB18551 & 598478 & 3999091 & 243.8 & 243.8 \\
\hline 63 & PB1880 & 590453 & 4006307 & 192.6 & 162.2 \\
\hline 64 & PB1900 & 586672 & 4012309 & 262.1 & 253 \\
\hline 65 & PB 19077 & 595712 & 3998290 & 106.7 & 105.5 \\
\hline 66 & PB1923 & 595029 & 4003552 & 326.1 & 324 \\
\hline 67 & PB1933 & 589554 & 4013601 & 261.2 & 228.6 \\
\hline 68 & PB 19508 & 588830 & 4003856 & 106.7 & 106.4 \\
\hline 69 & PB2109 & 589542 & 4014803 & 335.9 & 335.9 \\
\hline 70 & PB2114 & 588328 & 4016424 & 181.4 & 177.1 \\
\hline 71 & PB2135 & 596864 & 4000306 & 153.9 & 153.9 \\
\hline 72 & PB21572 & 592522 & 4006760 & 121.9 & 121.9 \\
\hline 73 & PB21613 & 591732 & 3998679 & 91.4 & 90.2 \\
\hline 74 & PB22211 & 587548 & 4007079 & 121.9 & 121.9 \\
\hline 75 & PB22728 & 588739 & 4015195 & 123.1 & 121.6 \\
\hline 76 & PB2308 & 596120 & 3997493 & 155.4 & 154.2 \\
\hline 77 & РB2309 & 580910 & 4006583 & 91.4 & 90.8 \\
\hline 78 & PB23093 & 590012 & 4007936 & 121.9 & 120.1 \\
\hline 79 & PB23480 & 589176 & 4009129 & 249.9 & 249.6 \\
\hline 80 & PB25276 & 598487 & 3998290 & 246.9 & 246.3 \\
\hline 81 & PB257 & 587098 & 4019678 & 227.4 & 227.4 \\
\hline 82 & PB2640 & 593707 & 3998700 & 152.7 & 150.3 \\
\hline 83 & PB26840 & 590445 & 4007139 & 91.4 & 91.4 \\
\hline 84 & PB26870 & 590903 & 4003908 & 152.4 & 152.1 \\
\hline
\end{tabular}


Table 1. Location and depth of Pahrump Valley boreholes - Continued.

[Data are compiled from the State of Nevada, Division of Water Resources, Well Driller's Reports;

Easting and northing are in UTM meters, using UTM zone 11, NAD 27 coordinates; corrected thickness is computed by subtracting from the total depth of the hole any intervals that lacked lithologic descriptions and intervals described as soil]

\begin{tabular}{|c|c|c|c|c|c|}
\hline Index & Well name & $\begin{array}{c}\text { Easting } \\
\text { (UTM m) }\end{array}$ & $\begin{array}{l}\text { Northing } \\
\text { (UTM m) }\end{array}$ & $\begin{array}{l}\text { Total depth } \\
\text { (m) }\end{array}$ & $\begin{array}{l}\text { Corrected } \\
\text { depth (m) }\end{array}$ \\
\hline 85 & PB26880 & 596245 & 4004366 & 106.7 & 105.5 \\
\hline 86 & PB2703 & 596830 & 4001137 & 180.4 & 172.8 \\
\hline 87 & PB2760 & 592507 & 3998687 & 106.7 & 104.0 \\
\hline 88 & PB27606 & 585448 & 4009862 & 96.0 & 96.0 \\
\hline 89 & PB27616 & 595012 & 4005154 & 153.9 & 153.9 \\
\hline 90 & PB27617 & 595012 & 4005154 & 153.9 & 153.9 \\
\hline 91 & PB27621 & 593345 & 3997494 & 100.0 & 100.0 \\
\hline 92 & PB2774 & 590445 & 4007139 & 119.5 & 119.5 \\
\hline 93 & PB28189 & 593345 & 3997494 & 160.0 & 60.0 \\
\hline 94 & PB28192 & 592926 & 4006364 & 121.9 & 121.9 \\
\hline 95 & PB28415 & 588311 & 4010723 & 226.2 & 226.2 \\
\hline 96 & PB28424 & 593246 & 4016105 & 281.9 & 281.9 \\
\hline 97 & PB2871 & 585868 & 4010298 & 112.8 & 101.8 \\
\hline 98 & PB29418 & 601652 & 3999097 & 104.9 & 100.6 \\
\hline 99 & PB29438 & 592865 & 4000293 & 91.4 & 90.2 \\
\hline 100 & PB29817 & 589134 & 4015631 & 182.9 & 182.9 \\
\hline 101 & PB2985 & 585068 & 4007855 & 91.4 & 60.9 \\
\hline 102 & PB3011 & 598068 & 3999918 & 100.6 & 100.6 \\
\hline 103 & PB3075 & 588360 & 4008320 & 91.4 & 91.4 \\
\hline 104 & PB30978 & 598839 & 4000328 & 91.4 & 91.4 \\
\hline 105 & PB3166 & 589126 & 4013997 & 163.7 & 161.9 \\
\hline 106 & PB32328 & 591328 & 4003913 & 274.3 & 274.3 \\
\hline 107 & PB32663 & 590441 & 4007540 & 91.4 & 90.2 \\
\hline 108 & PB32959 & 585900 & 4007062 & 91.4 & 53.3 \\
\hline 109 & PB3314 & 599291 & 3997898 & 153.0 & 153.0 \\
\hline 110 & PB33981 & 592030 & 4010792 & 109.7 & 108.5 \\
\hline 111 & PB33992 & 592047 & 4009159 & 91.4 & 90.2 \\
\hline 112 & PB3445 & 592848 & 4001895 & 91.4 & 89.9 \\
\hline 113 & PB35236 & 588747 & 4019294 & 103.6 & 103.6 \\
\hline 114 & PB35716 & 591193 & 4011985 & 91.4 & 91.4 \\
\hline 115 & PB3580 & 589180 & 4008729 & 198.1 & 176.5 \\
\hline 116 & PB36142 & 588348 & 4009521 & 106.7 & 49.1 \\
\hline 117 & PB3634 & 589134 & 4015631 & 149.4 & 148.2 \\
\hline 118 & PB36494 & 593400 & 3994660 & 91.4 & 42.6 \\
\hline 119 & PB3720 & 593623 & 4001903 & 100.6 & 97.3 \\
\hline 120 & PB37635 & 594473 & 3999509 & 91.4 & 91.4 \\
\hline 121 & PB37975 & 593269 & 3999897 & 91.4 & 91.4 \\
\hline 122 & PB38493 & 598496 & 3997489 & 91.4 & 91.4 \\
\hline 123 & PB3855 & 588315 & 4012756 & 155.4 & 79.2 \\
\hline 124 & PB40165 & 592454 & 4020164 & 353.6 & 353.6 \\
\hline 125 & PB4020 & 598078 & 3999087 & 139.0 & 131.4 \\
\hline
\end{tabular}


Table 1. Location and depth of Pahrump Valley boreholes - Continued.

[Data are compiled from the State of Nevada, Division of Water Resources, Well Driller's Reports;

Easting and northing are in UTM meters, using UTM zone 11, NAD 27 coordinates; corrected thickness is computed by subtracting from the total depth of the hole any intervals that lacked lithologic descriptions and intervals described as soil]

\begin{tabular}{|c|c|c|c|c|c|}
\hline Index & Well name & $\begin{array}{c}\text { Easting } \\
\text { (UTM m) }\end{array}$ & $\begin{array}{l}\text { Northing } \\
\text { (UTM m) }\end{array}$ & $\begin{array}{l}\text { Total depth } \\
\text { (m) }\end{array}$ & $\begin{array}{l}\text { Corrected } \\
\text { depth (m) }\end{array}$ \\
\hline 126 & PB4023 & 588166 & 3997810 & 140.2 & 120.4 \\
\hline 127 & PB41083 & 591185 & 4012817 & 99.1 & 99.1 \\
\hline 128 & PB41147 & 598473 & 3999492 & 124.4 & 124.4 \\
\hline 129 & PB41224 & 587518 & 4022517 & 143.3 & 137.8 \\
\hline 130 & PB41936 & 594174 & 4006378 & 97.5 & 97.5 \\
\hline 131 & PB41957 & 598473 & 3999492 & 216.4 & 92.0 \\
\hline 132 & PB44192 & 601652 & 3999097 & 109.7 & 109.7 \\
\hline 133 & PB44193 & 601652 & 3999097 & 109.7 & 109.7 \\
\hline 134 & PB44640 & 591185 & 4012817 & 99.1 & 99.1 \\
\hline 135 & PB44689 & 592051 & 4008758 & 105.2 & 105.2 \\
\hline 136 & PB4469 & 593767 & 3995465 & 121.9 & 68.6 \\
\hline 137 & PB4508 & 599225 & 4001534 & 219.5 & 214 \\
\hline 138 & PB45153 & 587530 & 4021315 & 114.3 & 114.3 \\
\hline 139 & PB45214 & 600077 & 3999109 & 91.4 & 91.4 \\
\hline 140 & PB4533 & 594625 & 4003948 & 249.9 & 243.8 \\
\hline 141 & PB46904 & 587858 & 4025817 & 292.6 & 292.6 \\
\hline 142 & PB47289 & 592462 & 4009995 & 120.7 & 120.7 \\
\hline 143 & PB47448 & 592475 & 4008763 & 106.7 & 106.7 \\
\hline 144 & PB47843 & 587090 & 4022944 & 151.5 & 151.5 \\
\hline 145 & PB47846 & 591600 & 4013622 & 116.4 & 116.4 \\
\hline 146 & PB4789 & 587488 & 4015583 & 106.7 & 103.7 \\
\hline 147 & PB4792 & 586682 & 4018841 & 140.2 & 138.4 \\
\hline 148 & PB4793 & 587102 & 4019246 & 234.1 & 227.1 \\
\hline 149 & PB4926 & 587504 & 4016415 & 106.7 & 82.9 \\
\hline 150 & PB50907 & 593400 & 3994660 & 91.4 & 88.4 \\
\hline 151 & PB5092 & 596873 & 3999505 & 152.4 & 141.7 \\
\hline 152 & PB51228 & 586662 & 4023340 & 157.0 & 157.0 \\
\hline 153 & PB51521 & 590924 & 3999471 & 121.9 & 120.7 \\
\hline 154 & PB52055 & 593308 & 4007971 & 111.3 & 111.3 \\
\hline 155 & PB52056 & 593342 & 4007170 & 103.6 & 103.6 \\
\hline 156 & PB53304 & 590370 & 4011976 & 91.4 & 84.7 \\
\hline 157 & PB5393 & 585859 & 4018833 & 143.3 & 140.3 \\
\hline 158 & PB5590 & 592964 & 4005132 & 238.7 & 238.7 \\
\hline 159 & PB58064 & 588756 & 4020927 & 152.4 & 152.4 \\
\hline 160 & PB5846 & 588327 & 4013989 & 155.4 & 155.4 \\
\hline 161 & PB5848 & 587496 & 4014782 & 152.4 & 152.4 \\
\hline 162 & PB58960 & 592471 & 4009194 & 103.6 & 103.6 \\
\hline 163 & PB60808 & 593342 & 4007170 & 96.0 & 96.0 \\
\hline 164 & PB61022 & 587094 & 4022512 & 131.1 & 120.4 \\
\hline 165 & PB6175 & 588311 & 4013157 & 157.0 & 157.0 \\
\hline 166 & PB61843 & 593266 & 4016506 & 233.2 & 233.2 \\
\hline
\end{tabular}


Table 1. Location and depth of Pahrump Valley boreholes - Continued.

[Data are compiled from the State of Nevada, Division of Water Resources, Well Driller's Reports;

Easting and northing are in UTM meters, using UTM zone 11, NAD 27 coordinates; corrected thickness is computed by subtracting from the total depth of the hole any intervals that lacked lithologic descriptions and intervals described as soil]

\begin{tabular}{|c|c|c|c|c|c|}
\hline Index & Well name & $\begin{array}{c}\text { Easting } \\
\text { (UTM m) }\end{array}$ & $\begin{array}{l}\text { Northing } \\
\text { (UTM m) }\end{array}$ & $\begin{array}{l}\text { Total depth } \\
\text { (m) }\end{array}$ & $\begin{array}{l}\text { Corrected } \\
\text { depth }(\mathrm{m})\end{array}$ \\
\hline 167 & PB61844 & 593246 & 4016105 & 306.3 & 306.3 \\
\hline 168 & PB62094 & 599032 & 4000915 & 104.2 & 103.0 \\
\hline 169 & PB62096 & 599225 & 4001534 & 100.6 & 99.3 \\
\hline 170 & PB62097 & 599634 & 4000737 & 91.4 & 91.4 \\
\hline 171 & PB62099 & 599634 & 4000737 & 91.4 & 87.7 \\
\hline 172 & PB62130 & 600034 & 4000742 & 91.4 & 67.0 \\
\hline 173 & PB62208 & 601652 & 3999097 & 115.2 & 115.2 \\
\hline 174 & PB62211 & 601652 & 3999097 & 115.8 & 115.8 \\
\hline 175 & PB62214 & 600482 & 3998682 & 115.2 & 115.2 \\
\hline 176 & PB6261 & 593665 & 4000302 & 97.5 & 96.3 \\
\hline 177 & PB62653 & 597668 & 3999914 & 122.5 & 121.3 \\
\hline 178 & PB62932 & 590374 & 4011576 & 121.9 & 110.9 \\
\hline 179 & PB63198 & 590819 & 4009547 & 153.9 & 153.9 \\
\hline 180 & PB63205 & 590844 & 4007143 & 182.9 & 175.9 \\
\hline 181 & PB63280 & 589184 & 4008328 & 91.4 & 91.4 \\
\hline 182 & PB63291 & 590012 & 4007936 & 91.4 & 91.4 \\
\hline 183 & PB6475 & 591332 & 3998675 & 121.9 & 121.9 \\
\hline 184 & PB64821 & 593750 & 4006373 & 91.4 & 91.4 \\
\hline 185 & PB6510 & 590012 & 4007936 & 201.8 & 182.0 \\
\hline 186 & PB65583 & 589982 & 4018074 & 248.4 & 248.4 \\
\hline 187 & PB68452 & 589612 & 4007932 & 91.4 & 91.4 \\
\hline 188 & PB68455 & 593750 & 4006373 & 91.4 & 91.4 \\
\hline 189 & PB69403 & 591616 & 4016889 & 306.3 & 306.3 \\
\hline 190 & PB6942 & 594473 & 3999509 & 144.8 & 144.8 \\
\hline 191 & PB69514 & 583839 & 4005810 & 310.9 & 310.9 \\
\hline 192 & PB69572 & 589184 & 4008328 & 121.9 & 121.9 \\
\hline 193 & PB69742 & 591185 & 4012817 & 99.4 & 99.4 \\
\hline 194 & PB69743 & 591185 & 4012817 & 108.2 & 108.2 \\
\hline 195 & PB70982 & 592043 & 4018927 & 210.3 & 210.3 \\
\hline 196 & PB7132 & 593801 & 4003939 & 201.8 & 201.8 \\
\hline 197 & PB7138 & 589205 & 4006294 & 182.9 & 182.9 \\
\hline 198 & PB72598 & 587098 & 4022112 & 121.9 & 121.9 \\
\hline 199 & PB72977 & 593342 & 4007170 & 91.4 & 91.4 \\
\hline 200 & PB73033 & 592467 & 4009595 & 100.6 & 100.6 \\
\hline 201 & PB73078 & 592903 & 3999092 & 91.4 & 91.4 \\
\hline 202 & PB73120 & 591605 & 4013222 & 114.3 & 114.3 \\
\hline 203 & PB73124 & 590805 & 4018113 & 114.3 & 114.3 \\
\hline 204 & PB73669 & 592047 & 4009159 & 96.6 & 96.6 \\
\hline 205 & PB73694 & 593750 & 4006373 & 109.7 & 109.7 \\
\hline 206 & PB73695 & 592051 & 4008758 & 100.6 & 100.6 \\
\hline 207 & PB73698 & 591282 & 4005915 & 129.5 & 129.5 \\
\hline
\end{tabular}


Table 1. Location and depth of Pahrump Valley boreholes - Continued.

[Data are compiled from the State of Nevada, Division of Water Resources, Well Driller's Reports;

Easting and northing are in UTM meters, using UTM zone 11, NAD 27 coordinates; corrected thickness is computed by subtracting from the total depth of the hole any intervals that lacked lithologic descriptions and intervals described as soil]

\begin{tabular}{|c|c|c|c|c|c|}
\hline Index & Well name & $\begin{array}{c}\text { Easting } \\
\text { (UTM m) }\end{array}$ & $\begin{array}{l}\text { Northing } \\
\text { (UTM m) }\end{array}$ & $\begin{array}{l}\text { Total depth } \\
\text { (m) }\end{array}$ & $\begin{array}{l}\text { Corrected } \\
\text { depth (m) }\end{array}$ \\
\hline 208 & PB738 & 589126 & 4011563 & 135.6 & 135.6 \\
\hline 209 & PB7398 & 592865 & 4000293 & 139.0 & 139.0 \\
\hline 210 & PB7436 & 587526 & 4019250 & 137.2 & 137.2 \\
\hline 211 & PB7477 & 592907 & 3998691 & 236.2 & 236.2 \\
\hline 212 & PB7490 & 592941 & 3997890 & 152.4 & 152.4 \\
\hline 213 & PB75057 & 593342 & 4007170 & 106.7 & 106.7 \\
\hline 214 & PB7515 & 590403 & 4008741 & 114.3 & 114.3 \\
\hline 215 & PB7516 & 591282 & 4001078 & 120.4 & 120.4 \\
\hline 216 & PB7695 & 591737 & 3998278 & 121.9 & 121.9 \\
\hline 217 & PB7740 & 590474 & 4001870 & 182.9 & 182.9 \\
\hline 218 & PB7750 & 587936 & 4008315 & 121.9 & 121.9 \\
\hline 219 & PB7833 & 596069 & 3999896 & 167.6 & 167.6 \\
\hline 220 & PB7933 & 592141 & 3997882 & 138.7 & 138.7 \\
\hline 221 & PB7934 & 592082 & 4001086 & 105.2 & 105.2 \\
\hline 222 & PB8263 & 593707 & 3998700 & 149.4 & 149.4 \\
\hline 223 & PB829 & 594192 & 4004745 & 242.3 & 242.3 \\
\hline 224 & PB8339 & 588328 & 4016424 & 246.3 & 246.3 \\
\hline 225 & PB8356 & 590516 & 4000268 & 128.0 & 128.0 \\
\hline 226 & PB8438 & 587098 & 4022112 & 128.3 & 128.3 \\
\hline 227 & PB8579 & 586708 & 4008673 & 121.9 & 121.9 \\
\hline 228 & PB8587 & 593809 & 3993863 & 128.0 & 128.0 \\
\hline 229 & PB8705 & 592950 & 3997058 & 97.5 & 97.5 \\
\hline 230 & PB8750 & 592090 & 4000285 & 167.6 & 167.6 \\
\hline 231 & PB884 & 598496 & 3997489 & 259.4 & 259.4 \\
\hline 232 & PB8949 & 587965 & 4007884 & 121.9 & 121.9 \\
\hline 233 & PB8976 & 592145 & 3997450 & 121.9 & 121.9 \\
\hline 234 & PB9004 & 593042 & 3993053 & 121.9 & 121.9 \\
\hline 235 & PB9067 & 592132 & 3998683 & 134.1 & 134.1 \\
\hline 236 & PB9180 & 592903 & 3999092 & 121.9 & 121.9 \\
\hline 237 & PB9214 & 598491 & 3997889 & 290.2 & 290.2 \\
\hline 238 & PB9231 & 590933 & 3998670 & 121.9 & 121.9 \\
\hline 239 & PB9238 & 591274 & 4001879 & 105.2 & 105.2 \\
\hline 240 & PB9273 & 592238 & 3993446 & 152.4 & 152.4 \\
\hline 241 & PB9274 & 588360 & 4008320 & 128.6 & 128.6 \\
\hline 242 & PB9278 & 592600 & 3994651 & 121.9 & 121.9 \\
\hline 243 & PB9284 & 593843 & 3993062 & 121.9 & 121.9 \\
\hline 244 & PB9324 & 593042 & 3993053 & 152.4 & 152.4 \\
\hline 245 & PB9366 & 590479 & 4001470 & 92.4 & 92.4 \\
\hline 246 & PB9472 & 592106 & 4005924 & 147.8 & 147.8 \\
\hline 247 & PB9474 & 588377 & 4006687 & 160.0 & 160.0 \\
\hline 248 & PB9679 & 592541 & 3997886 & 98.1 & 98.1 \\
\hline 249 & PB9697 & 591291 & 4000276 & 93.0 & 93.0 \\
\hline
\end{tabular}


Table 1. Location and depth of Pahrump Valley boreholes - Continued.

[Data are compiled from the State of Nevada, Division of Water Resources, Well Driller's Reports; Easting and northing are in UTM meters, using UTM zone 11, NAD 27 coordinates; corrected thickness is computed by subtracting from the total depth of the hole any intervals that lacked lithologic descriptions and intervals described as soil]

\begin{tabular}{cccccc}
\hline Index & Well name & $\begin{array}{c}\text { Easting } \\
(\text { UTM m })\end{array}$ & $\begin{array}{c}\text { Northing } \\
(\text { UTM m })\end{array}$ & $\begin{array}{c}\text { Total depth } \\
(\mathrm{m})\end{array}$ & $\begin{array}{c}\text { Corrected } \\
\text { depth }(\mathrm{m})\end{array}$ \\
\hline 250 & PB9795 & 593299 & 3999496 & 121.9 & 121.9 \\
251 & PB9968 & 589953 & 4013605 & 152.4 & 152.4 \\
252 & PB9969 & 592907 & 3998691 & 104.2 & 104.2 \\
253 & PB8424 & 603334 & 3992182 & 121.9 & 120.7 \\
254 & PB8749 & 603369 & 3991351 & 99.1 & 97.2 \\
255 & PB29095 & 596925 & 3997070 & 97.5 & 97.5 \\
256 & PB9475 & 595325 & 3997083 & 147.8 & 74.0 \\
257 & PB10806 & 594662 & 3995967 & 121.9 & 121.9 \\
258 & PB10850 & 594158 & 3996270 & 137.2 & 137.2 \\
259 & PB62403 & 595358 & 3996283 & 60.9 & 60.7 \\
260 & PB48671 & 594563 & 3995873 & 54.9 & 53.6 \\
261 & PB52765 & 594925 & 3997079 & 57.9 & 57.9 \\
262 & PB4213 & 595307 & 3998686 & 160.0 & 146.3 \\
263 & PB50732 & 605692 & 3997880 & 204.2 & 204.2 \\
264 & PB62542 & 610332 & 3992761 & 152.4 & 152.4 \\
265 & PB61021 & 592468 & 4030549 & 272.5 & 261.8 \\
266 & PB46904 & 587858 & 4025817 & 289.6 & 289.6 \\
267 & 02DS04 & 599432 & 4010025 & 12.3 & 12.3 \\
268 & (outcrop) & & & & 24.0 \\
269 & 02DS05 & 602098 & 4013373 & 24.0 & 39.0 \\
270 & (outcrop) & & & 39.0 & 35.0 \\
\hline & (outcrob) & 601851 & 4013146 & 35.0 &
\end{tabular}

Data acquired from drilling records require tedious filtering to provide descriptions that are consistent and geologically meaningful. Lithologic descriptions were simplified into one of 14 categories (table 2). This process is by necessity interpretive; each field description is interpreted with the knowledge of the geologic units mapped at the surface and the characteristics of the surrounding data. For many of the records, described intervals could easily be assigned to one of the 14 lithologic descriptors, but in certain cases, lithologic descriptions may have been inadequate to make a proper assignment. In other instances, it is possible that more than one lithologic descriptor may have been appropriate. In the absence of cuttings, grain size information is subjective and does not necessarily correspond to standard grain size definitions. Lithologic descriptors were kept as generic as possible; stratigraphic names or names with genetic significance were avoided. One aspect of the lithologic description that was ignored because of the inconsistency of the driller's descriptions was the degree of induration of the sediment. Thus, "gravel" was not distinguished from "conglomerate"; "shale" was not distinguished from "clay". The resulting set of 266 holes contained about 3900 intervals where a geologist was required to make decisions regarding the lithologic identity of the interval. Stratigraphic sections created from multiple borehole logs (fig. 3) show a general consistency in lithologic trends. 
Table 2. Lithologic descriptors derived from borehole logs

[Driller's logs were obtained from State of Nevada: State of Nevada, Division of Water Resources, Well Driller's Report]

\begin{tabular}{|c|c|}
\hline Lithologic descriptor & Corresponding descriptions from driller's logs \\
\hline Conglomerate & $\begin{array}{l}\text { boulders; boulders \& gravel; cement gravel; rock \& cement gravel; } \\
\text { cemented boulders; cemented gravel; gravel; conglomerate; cemented } \\
\text { conglomerate rock; cemented gravel \& boulders; cemented rocks \& } \\
\text { gravel; cemented sand \& gravel; cobbles; lime cement gravel; loose } \\
\text { gravel }\end{array}$ \\
\hline Conglomerate and mudstone & $\begin{array}{l}\text { clay \& gravel; brown clay mixed with some gravel; clay \& boulders; clay } \\
\& \text { rock; cobbles clay; gravel with clay streaks }\end{array}$ \\
\hline Sand and gravel & $\begin{array}{l}\text { sand \& gravel; cemented rock \& sand; coarse sand fine gravel; gravel } \\
\text { boulders sand water; loose cobble \& sand; sand gravel \& cobbles }\end{array}$ \\
\hline Sandstone & $\begin{array}{l}\text { sand water; cemented sand; coarse sand; fine dark sand; hard sand; hard } \\
\text { sand \& rock; quartz; quick sand; sand; sand stone }\end{array}$ \\
\hline Siltstone & Silt; silty clay \\
\hline Mudstone & $\begin{array}{l}\text { bentonite clay; brown clay; clay; hard clay formation; red shale; soft } \\
\text { clay; sticky brown clay; lime shale; red shale \& limestone; sandy clay } \\
40 \% \text { caliche }\end{array}$ \\
\hline Sand Mudstone & $\begin{array}{l}\text { brown sandy clay; clay \& sand; hard shale \& sand water; sand \& clay; } \\
\text { sandstone with shale; sandy clay with gravel }\end{array}$ \\
\hline Mudstone and limestone & brown clay \& caliche; clay with streaks of caliche; lime \& clay \\
\hline Silty limestone & sandy formation with white rock; sandy lime; sandy loose clay \& caliche \\
\hline Gypsum & gypsum formation \\
\hline Limestone & $\begin{array}{l}\text { caliche; caliche \& limestone; cemented limestone; fractured limestone; } \\
\text { hard brown caliche; hard calcite; hard gray lime; hard lime shell; hard } \\
\text { white layer; lime; limestone; soft white rock formation }\end{array}$ \\
\hline Basalt & black rock; black volcanic rock; fractured black rock; \\
\hline nd & $\begin{array}{l}\text { no data; formation unknown; hard formation; ???; cemented gray small } \\
\text { trace clay; rock; [blank entry] }\end{array}$ \\
\hline Soil & $\begin{array}{l}\text { sandy loam; sandy surface; soil; surface; surface soil; top soil; top soil \& } \\
\text { rock }\end{array}$ \\
\hline
\end{tabular}

\section{Outcrop data}

Alluvial material of the Pahrump fan is exposed in outcrop in the deeply incised Wheeler Wash, to the east of the Pahrump Valley (fig. 2; see also Malmberg, 1967, fig. 3); outcrops were observed in four localities (fig. 2). These outcrops consist of cliff faces 10-30 $\mathrm{m}$ high that expose rocks that have been mapped as older fanglomerate and alluvial fan material (Malberg, 1967; dePolo and others, 1999, Workman and others, 2002). The four observed outcrops all consisted of poorly stratified matrix to clast-supported conglomerate with subordinate thin stringers of finer-grained material. 


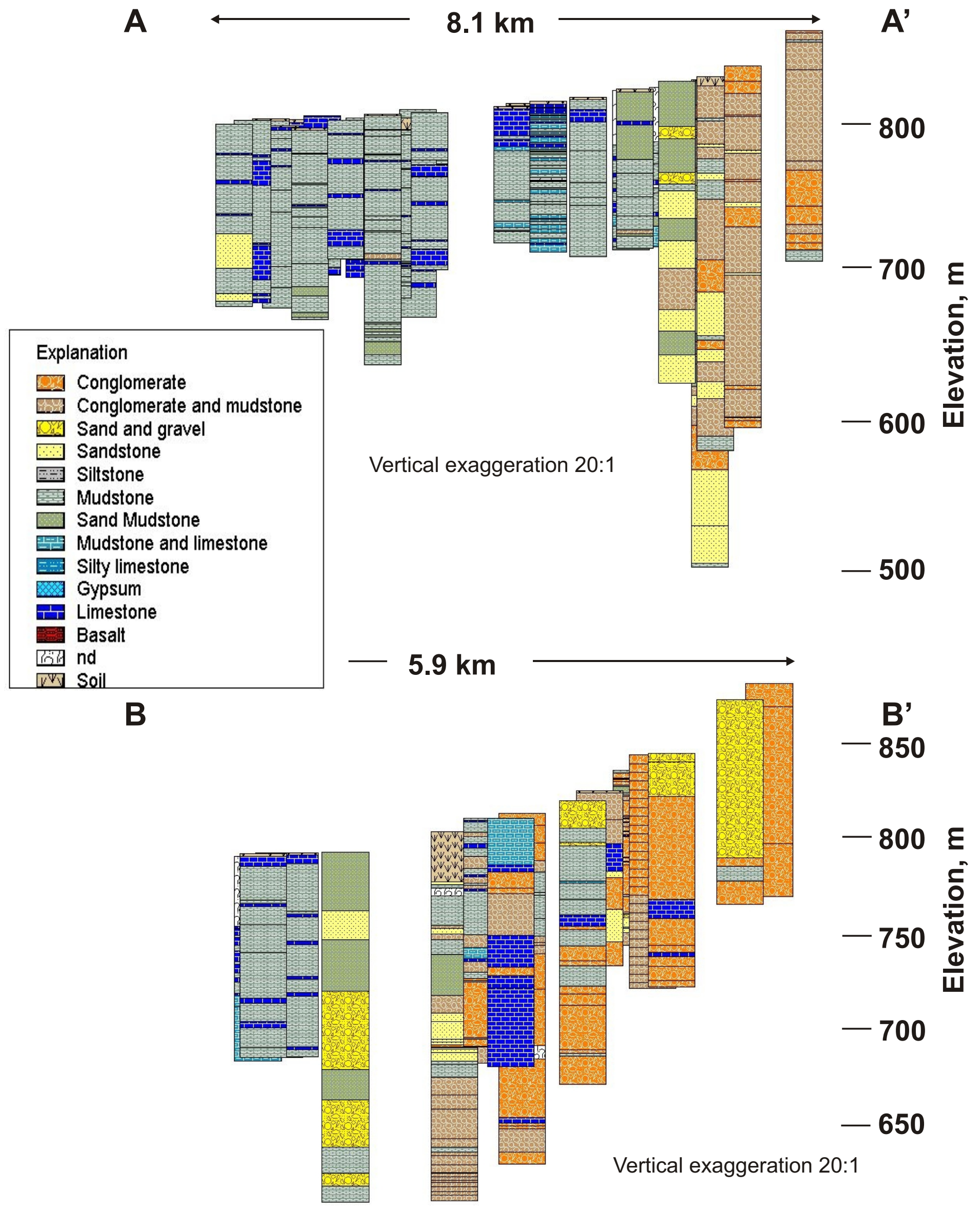

Figure 3. Example stratigraphic sections from the Pahrump Valley. Locations of sections are shown in figure 2 . All wells within $1 \mathrm{~km}$ of the section are projected onto the line of section. 
Lithologic data collected at each outcrop were collected so as to be as comparable as possible to the driller's logs from boreholes. Table 3 presents the outcrop data, including a lithologic descriptor that most closely represents what this material would have been called in a driller's log, plus generalized grain-sized information for both the conglomeratic material and the finer-grained interbeds.

Table 3. Lithologic descriptors derived from outcrops in Wheeler Wash

\begin{tabular}{ccccc}
\hline Name & $\begin{array}{c}\text { Lithologic } \\
\text { description } \\
\text { Conglomerate }\end{array}$ & $\begin{array}{c}\text { Clast size in coarse fan } \\
\text { material }\end{array}$ & $\begin{array}{c}\text { Clast size in fine } \\
\text { grained interbeds } \\
02 \text { cms04 average } 5 \mathrm{~cm}\end{array}$ & $\begin{array}{c}\text { Distance from toe } \\
\text { of fan }(\mathrm{km}) \\
7.4\end{array}$ \\
02DS05 & Conglomerate & $1-10 \mathrm{~cm}$, average $4 \mathrm{~cm}$ & $0.5-2 \mathrm{~cm}$, average $1 \mathrm{~cm}$ & 11.3 \\
02DS06 & Conglomerate & $2-4 \mathrm{~cm}$, average $3 \mathrm{~cm}$ & $\begin{array}{l}0.2-1 \mathrm{~cm} \text { clasts in sand- } \\
\text { sized matrix } \\
0.5 \mathrm{~cm} \text { to sand }\end{array}$ & 11.6 \\
02DS07 & Sand and gravel & $1-5 \mathrm{~cm}$, average $2 \mathrm{~cm}$ & 15.3 \\
\hline
\end{tabular}

\section{Calculations}

From the above data, the following aggregate thicknesses were computed for all of the lithologic intervals in each borehole and outcrop locality (table 4):

Corrected Thickness: This value was computed by subtracting from the total depth of the hole any intervals that lacked lithologic descriptions and intervals described as soil in the uppermost portions of the hole. The resulting corrected thickness represents those intervals of the hole that penetrated bedrock units for which lithologic descriptions were available.

Thickness of coarse grained material: The total thickness of coarse grained material in each hole was calculated as the aggregate thickness of all lithologic intervals described as conglomerate, sand and gravel, sandstone, or tuffaceous sandstone.

Thickness of fine grained material: The total thickness of fine grained material in each hole was calculated as the aggregate thickness of all lithologic intervals described as conglomerate and mudstone, siltstone, mudstone, sand mudstone, or mudstone and limestone.

Thickness of limestone: The total thickness of carbonate material in each hole was calculated as the aggregate thickness of all lithologic intervals described as limestone or silty limestone.

Thickness of volcanic material: The total thickness of volcanic material was calculated as the aggregate thickness of all lithologic intervals described as tuff, welded tuff, or basalt. In the Pahrump Valley, these descriptors only applied to less than ten wells.

From these thickness data, the following percentages were computed for each borehole and outcrop locality (table 4):

Percent sedimentary rock component is calculated as (Thickness of coarse grained material+ Thickness of fine grained material+ Thickness of limestone)/Corrected thickness (fig. 4); 
Percent coarse grained material in the sedimentary component is calculated as

(Thickness of coarse grained material)

(Thickness of coarse grained material+ Thickness of fine grained material+ Thickness of limestone)

\section{Explanation of units}

Soil

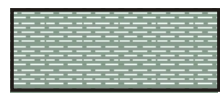

Mudstone

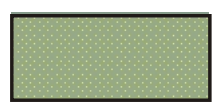

Sandy mudstone

Conglomerate
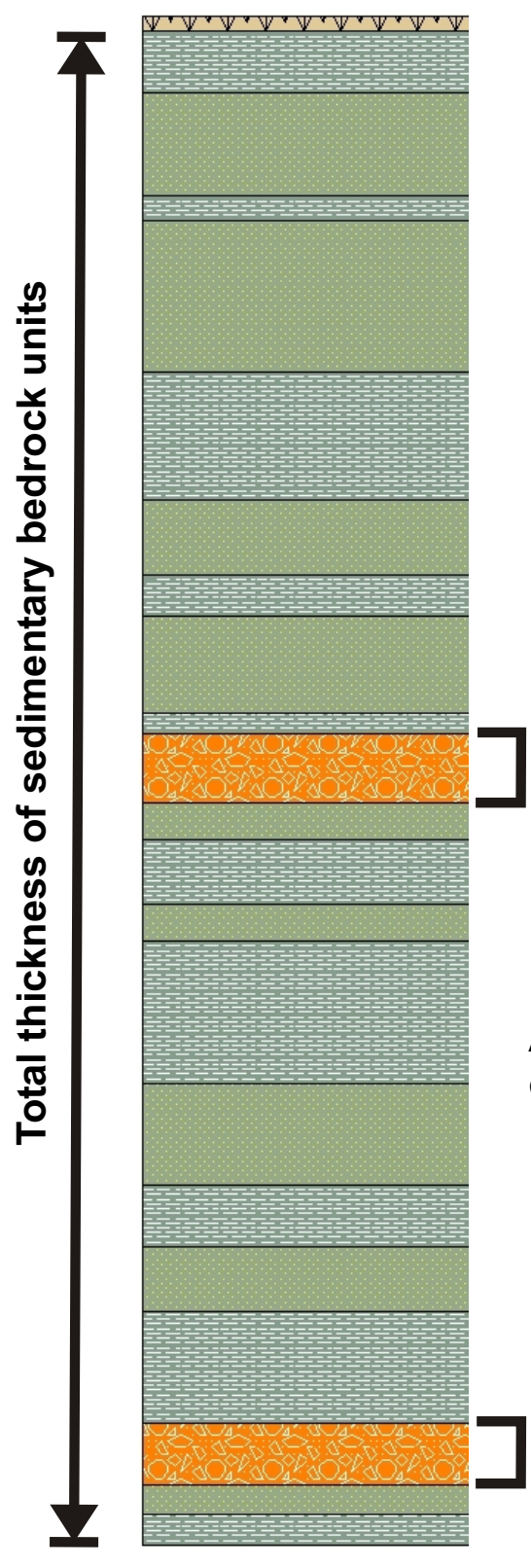

Aggregate thickness of conglomerate

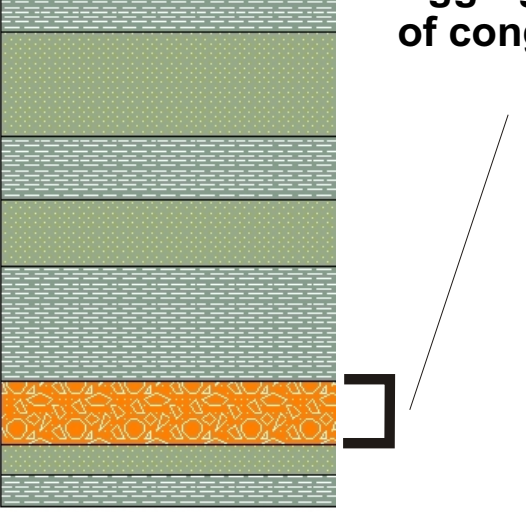

Figure 4. Sample borehole showing methodology for calculation of coarse grained sedimentary component. 
Percent volcanic rock component is calculated as Thickness of volcanic material/corrected thickness;

Percent limestone is calculated as (limestone thickness/corrected thickness);

Percent coarse grained material in the sedimentary component is calculated as (Thickness of coarse grained material /(Thickness of coarse grained material+ Thickness of fine grained material+ Thickness of limestone).

Table 4. Lithologic data from Pahrump Valley boreholes

[Data are compiled from the State of Nevada, Division of Water Resources, Well Driller's Reports]

\begin{tabular}{|c|c|c|c|c|c|c|c|c|}
\hline Index & $\begin{array}{l}\text { Thickness } \\
\text { of } \\
\text { volcanic } \\
\text { rock } \\
\text { (m) }\end{array}$ & $\begin{array}{l}\text { Thickness of } \\
\text { coarse grained } \\
\text { sedimentary } \\
\text { rocks }(\mathrm{m})\end{array}$ & $\begin{array}{l}\text { Thickness of } \\
\text { fine grained } \\
\text { sedimentary } \\
\text { rocks } \\
\text { (m) }\end{array}$ & $\begin{array}{c}\text { Limestone } \\
\text { thickness } \\
\text { (m) }\end{array}$ & $\begin{array}{l}\text { Percent } \\
\text { Volcanic } \\
\text { rocks }\end{array}$ & $\begin{array}{c}\text { Percent } \\
\text { Sedimentary } \\
\text { rocks }\end{array}$ & $\begin{array}{c}\text { Percent } \\
\text { limestone }\end{array}$ & $\begin{array}{c}\text { Percent } \\
\text { coarse } \\
\text { grained } \\
\text { sedimentary } \\
\text { rocks }\end{array}$ \\
\hline 1 & 0 & 0 & 85.6 & 3.7 & 0 & 100 & 4.1 & 0 \\
\hline 2 & 0 & 0.6 & 105.9 & 4.8 & 0 & 100 & 4.3 & 0.5 \\
\hline 3 & 0 & 0 & 84.7 & 8.9 & 0 & 100 & 9.5 & 0 \\
\hline 4 & 0 & 199.6 & 0 & 0 & 0 & 100 & 0 & 100 \\
\hline 5 & 0 & 24.3 & 128.7 & 1.5 & 0 & 100 & 1 & 15.7 \\
\hline 6 & 0 & 7.7 & 73.7 & 9.2 & 0 & 100 & 10.2 & 8.5 \\
\hline 7 & 0 & 28.1 & 75.7 & 21.2 & 0 & 100 & 17 & 22.5 \\
\hline 8 & 0 & 33 & 78.2 & 35.5 & 0 & 100 & 24.2 & 22.5 \\
\hline 9 & 0 & 3.3 & 98.2 & 27.4 & 0 & 100 & 21.3 & 2.6 \\
\hline 10 & 0 & 0 & 86.7 & 22.4 & 0 & 100 & 20.5 & 0 \\
\hline 11 & 0 & 0 & 85.7 & 35.6 & 0 & 100 & 29.3 & 0 \\
\hline 12 & 0 & 1.2 & 86.5 & 3.1 & 0 & 100 & 3.4 & 1.3 \\
\hline 13 & 0 & 0 & 121.7 & 8.5 & 0 & 100 & 6.5 & 0 \\
\hline 14 & 0 & 1.9 & 87.8 & 7.9 & 0 & 100 & 8.1 & 1.9 \\
\hline 15 & 0 & 0 & 90.9 & 26.4 & 0 & 100 & 22.5 & 0 \\
\hline 16 & 0 & 0 & 113.3 & 7.4 & 0 & 100 & 6.1 & 0 \\
\hline 17 & 0 & 0 & 82.1 & 40.8 & 0 & 100 & 33.2 & 0 \\
\hline 18 & 0 & 0 & 69.1 & 36.7 & 0 & 100 & 34.7 & 0 \\
\hline 19 & 0 & 0 & 51.7 & 54.7 & 0 & 100 & 51.4 & 0 \\
\hline 20 & 0 & 142 & 79.6 & 0 & 0 & 100 & 0 & 64.1 \\
\hline 21 & 0 & 0 & 48.2 & 21.9 & 0 & 100 & 31.2 & 0 \\
\hline 22 & 0 & 6.1 & 29.3 & 34.7 & 0 & 100 & 49.5 & 8.7 \\
\hline 23 & 0 & 0 & 54 & 16.1 & 0 & 100 & 23 & 0 \\
\hline 24 & 0 & 0 & 62.7 & 5.5 & 0 & 100 & 8.1 & 0 \\
\hline 25 & 0 & 0 & 56.5 & 13.6 & 0 & 100 & 19.4 & 0 \\
\hline 26 & 0 & 51.8 & 85.4 & 0 & 0 & 100 & 0 & 37.8 \\
\hline 27 & 0 & 2.5 & 76.1 & 11.6 & 0 & 100 & 12.9 & 2.8 \\
\hline 28 & 0 & 0 & 96.6 & 8.9 & 0 & 100 & 8.4 & 0 \\
\hline 29 & 0 & 2.1 & 90.9 & 0 & 0 & 100 & 0 & 2.3 \\
\hline 30 & 32.2 & 22.3 & 0.9 & 0 & 58.1 & 41.9 & 0 & 96.1 \\
\hline 31 & 0 & 9.5 & 111.6 & 5.7 & 0 & 100 & 4.5 & 7.5 \\
\hline
\end{tabular}


Table 4. Lithologic data from Pahrump Valley boreholes - Continued.

[Data are compiled from the State of Nevada, Division of Water Resources, Well Driller's Reports]

\begin{tabular}{|c|c|c|c|c|c|c|c|c|}
\hline Index & $\begin{array}{l}\text { Thickness } \\
\text { of } \\
\text { volcanic } \\
\text { rock } \\
\text { (m) }\end{array}$ & $\begin{array}{c}\text { Thickness of } \\
\text { coarse grained } \\
\text { sedimentary rocks } \\
(\mathrm{m})\end{array}$ & $\begin{array}{l}\text { Thickness of } \\
\text { fine grained } \\
\text { sedimentary } \\
\text { rocks } \\
\text { (m) }\end{array}$ & $\begin{array}{l}\text { Limestone } \\
\text { thickness } \\
\text { (m) }\end{array}$ & $\begin{array}{c}\text { Percent } \\
\text { Volcanic } \\
\text { rocks }\end{array}$ & $\begin{array}{c}\text { Percent } \\
\text { Sedimentary } \\
\text { rocks }\end{array}$ & $\begin{array}{c}\text { Percent } \\
\text { limestone }\end{array}$ & $\begin{array}{c}\text { Percent } \\
\text { coarse } \\
\text { grained } \\
\text { sedimentary } \\
\text { rocks }\end{array}$ \\
\hline 32 & 0 & 2.7 & 138 & 42.2 & 0 & 100 & 23.1 & 1.5 \\
\hline 33 & 0 & 171.4 & 45.6 & 0 & 0 & 100 & 0 & 79 \\
\hline 34 & 2.7 & 0 & 126.3 & 23.4 & 1.8 & 98.2 & 15.4 & 0 \\
\hline 35 & 0 & 1.2 & 87.2 & 35.6 & 0 & 100 & 28.7 & 1 \\
\hline 36 & 0 & 82.8 & 8.6 & 0 & 0 & 100 & 0 & 90.6 \\
\hline 37 & 0 & 0 & 91.6 & 13.9 & 0 & 100 & 13.2 & 0 \\
\hline 38 & 0 & 24.4 & 74.4 & 0 & 0 & 100 & 0 & 24.7 \\
\hline 39 & 0 & 0 & 93 & 12.5 & 0 & 100 & 11.8 & 0 \\
\hline 40 & 0 & 0 & 92.8 & 12.4 & 0 & 100 & 11.8 & 0 \\
\hline 41 & 0 & 69 & 21.8 & 0.6 & 0 & 100 & 0.7 & 75.5 \\
\hline 42 & 0 & 0 & 41.4 & 49.7 & 0 & 100 & 54.6 & 0 \\
\hline 43 & 0 & 49.6 & 26.8 & 14.1 & 0 & 100 & 15.6 & 54.8 \\
\hline 44 & 0 & 0 & 167.6 & 0 & 0 & 100 & 0 & 0 \\
\hline 45 & 0 & 68.2 & 21.4 & 0 & 0 & 100 & 0 & 76.1 \\
\hline 46 & 0 & 0 & 88.1 & 2.7 & 0 & 100 & 3 & 0 \\
\hline 47 & 0 & 0 & 152.4 & 0 & 0 & 100 & 0 & 0 \\
\hline 48 & 0 & 0 & 86.5 & 3.1 & 0 & 100 & 3.5 & 0 \\
\hline 49 & 0 & 0 & 97.2 & 8 & 0 & 100 & 7.6 & 0 \\
\hline 50 & 0 & 0 & 101.8 & 2.5 & 0 & 100 & 2.4 & 0 \\
\hline 51 & 0 & 0.6 & 148.6 & 0.7 & 0 & 100 & 0.5 & 0.4 \\
\hline 52 & 0 & 7.9 & 82.9 & 0 & 0 & 100 & 0 & 8.7 \\
\hline 53 & 0 & 39.1 & 165.1 & 0 & 0 & 100 & 0 & 19.1 \\
\hline 54 & 0 & 0 & 89.9 & 0.6 & 0 & 100 & 0.7 & 0 \\
\hline 55 & 0 & 0 & 146.3 & 6.1 & 0 & 100 & 4 & 0 \\
\hline 56 & 0 & 9.4 & 85.4 & 0 & 0 & 100 & 0 & 9.9 \\
\hline 57 & 0 & 5.2 & 116.4 & 0 & 0 & 100 & 0 & 4.3 \\
\hline 58 & 0 & 2.1 & 78.7 & 7.6 & 0 & 100 & 8.6 & 2.4 \\
\hline 59 & 0 & 54.7 & 162 & 0 & 0 & 100 & 0 & 25.2 \\
\hline 60 & 0 & 51.5 & 152.7 & 0 & 0 & 100 & 0 & 25.2 \\
\hline 61 & 0 & 121.6 & 112.8 & 0 & 0 & 100 & 0 & 51.9 \\
\hline 62 & 0 & 52.7 & 191.1 & 0 & 0 & 100 & 0 & 21.6 \\
\hline 63 & 0 & 29.5 & 132.7 & 0 & 0 & 100 & 0 & 18.2 \\
\hline 64 & 0 & 26.5 & 216.2 & 10.3 & 0 & 100 & 4.1 & 10.5 \\
\hline 65 & 0 & 2.4 & 103.1 & 0 & 0 & 100 & 0 & 2.3 \\
\hline 66 & 0 & 178.7 & 145.3 & 0 & 0 & 100 & 0 & 55.2 \\
\hline 67 & 0 & 54.2 & 154.3 & 20.1 & 0 & 100 & 8.8 & 23.7 \\
\hline 68 & 0 & 3.6 & 90.8 & 12 & 0 & 100 & 11.3 & 3.4 \\
\hline 69 & 0 & 212.9 & 123 & 0 & 0 & 100 & 0 & 63.4 \\
\hline
\end{tabular}


Table 4. Lithologic data from Pahrump Valley boreholes - Continued.

[Data are compiled from the State of Nevada, Division of Water Resources, Well Driller's Reports]

\begin{tabular}{|c|c|c|c|c|c|c|c|c|}
\hline Index & $\begin{array}{l}\text { Thickness } \\
\text { of } \\
\text { volcanic } \\
\text { rock } \\
\text { (m) }\end{array}$ & $\begin{array}{c}\text { Thickness of } \\
\text { coarse grained } \\
\text { sedimentary rocks } \\
(\mathrm{m})\end{array}$ & $\begin{array}{l}\text { Thickness of } \\
\text { fine grained } \\
\text { sedimentary } \\
\text { rocks } \\
\text { (m) }\end{array}$ & $\begin{array}{l}\text { Limestone } \\
\text { thickness } \\
(\mathrm{m})\end{array}$ & $\begin{array}{l}\text { Percent } \\
\text { Volcanic } \\
\text { rocks }\end{array}$ & $\begin{array}{l}\text { Percent } \\
\text { Sedimentary } \\
\text { rocks }\end{array}$ & $\begin{array}{c}\text { Percent } \\
\text { limestone }\end{array}$ & $\begin{array}{c}\text { Percent } \\
\text { coarse } \\
\text { grained } \\
\text { sedimentary } \\
\text { rocks }\end{array}$ \\
\hline 70 & 0 & 61.9 & 112.8 & 2.4 & 0 & 100 & 1.4 & 35 \\
\hline 71 & 0 & 0 & 153.9 & 0 & 0 & 100 & 0 & 0 \\
\hline 72 & 0 & 77.7 & 44.2 & 0 & 0 & 100 & 0 & 63.7 \\
\hline 73 & 0 & 0 & 81.6 & 8.6 & 0 & 100 & 9.5 & 0 \\
\hline 74 & 0 & 22.2 & 99.7 & 0 & 0 & 100 & 0 & 18.2 \\
\hline 75 & 0 & 0 & 121.6 & 0 & 0 & 100 & 0 & 0 \\
\hline 76 & 0 & 39.6 & 114.6 & 0 & 0 & 100 & 0 & 25.7 \\
\hline 77 & 0 & 31.5 & 59.3 & 0 & 0 & 100 & 0 & 34.7 \\
\hline 78 & 0 & 15 & 102.7 & 2.4 & 0 & 100 & 2 & 12.5 \\
\hline 79 & 0 & 92 & 152.1 & 5.5 & 0 & 100 & 2.2 & 36.9 \\
\hline 80 & 0 & 22.1 & 224.2 & 0 & 0 & 100 & 0 & 9 \\
\hline 81 & 0 & 213.4 & 14 & 0 & 0 & 100 & 0 & 93.8 \\
\hline 82 & 0 & 0 & 90.5 & 59.8 & 0 & 100 & 39.8 & 0 \\
\hline 83 & 0 & 12.2 & 76.2 & 3 & 0 & 100 & 3.3 & 13.3 \\
\hline 84 & 0 & 0 & 135 & 17.1 & 0 & 100 & 11.2 & 0 \\
\hline 85 & 0 & 105.5 & 0 & 0 & 0 & 100 & 0 & 100 \\
\hline 86 & 0 & 3 & 168.9 & 0.9 & 0 & 100 & 0.5 & 1.7 \\
\hline 87 & 0 & 0 & 82.1 & 21.9 & 0 & 100 & 21.1 & 0 \\
\hline 88 & 0 & 16.8 & 71.9 & 7.3 & 0 & 100 & 7.6 & 17.5 \\
\hline 89 & 0 & 48.7 & 105.2 & 0 & 0 & 100 & 0 & 31.6 \\
\hline 90 & 0 & 48.7 & 105.2 & 0 & 0 & 100 & 0 & 31.6 \\
\hline 91 & 0 & 0 & 40.6 & 59.4 & 0 & 100 & 59.4 & 0 \\
\hline 92 & 0 & 33.3 & 79.3 & 6.9 & 0 & 100 & 5.8 & 27.9 \\
\hline 93 & 0 & 0 & 42.8 & 17.2 & 0 & 100 & 28.7 & 0 \\
\hline 94 & 0 & 109.6 & 0 & 12.3 & 0 & 100 & 10.1 & 89.9 \\
\hline 95 & 0 & 50.3 & 141.1 & 34.8 & 0 & 100 & 15.4 & 22.2 \\
\hline 96 & 0 & 281.9 & 0 & 0 & 0 & 100 & 0 & 100 \\
\hline 97 & 0 & 0 & 96.2 & 5.6 & 0 & 100 & 5.5 & 0 \\
\hline 98 & 0 & 100.6 & 0 & 0 & 0 & 100 & 0 & 100 \\
\hline 99 & 0 & 0 & 76.2 & 14 & 0 & 100 & 15.5 & 0 \\
\hline 100 & 0 & 62.8 & 98.6 & 21.5 & 0 & 100 & 11.8 & 34.3 \\
\hline 101 & 0 & 0 & 54.4 & 6.5 & 0 & 100 & 10.7 & 0 \\
\hline 102 & 0 & 9.7 & 87.3 & 3.6 & 0 & 100 & 3.6 & 9.6 \\
\hline 103 & 0 & 12.2 & 67.1 & 12.1 & 0 & 100 & 13.2 & 13.3 \\
\hline 104 & 0 & 18.1 & 58 & 15.3 & 0 & 100 & 16.7 & 19.8 \\
\hline
\end{tabular}


Table 4. Lithologic data from Pahrump Valley boreholes - Continued.

[Data are compiled from the State of Nevada, Division of Water Resources, Well Driller's Reports]

\begin{tabular}{|c|c|c|c|c|c|c|c|c|}
\hline Index & $\begin{array}{l}\text { Thickness } \\
\text { of } \\
\text { volcanic } \\
\text { rock } \\
\text { (m) }\end{array}$ & $\begin{array}{c}\text { Thickness of } \\
\text { coarse grained } \\
\text { sedimentary rocks } \\
\text { (m) }\end{array}$ & $\begin{array}{l}\text { Thickness of } \\
\text { fine grained } \\
\text { sedimentary } \\
\text { rocks } \\
\text { (m) }\end{array}$ & $\begin{array}{l}\text { Limestone } \\
\text { thickness } \\
(\mathrm{m})\end{array}$ & $\begin{array}{l}\text { Percent } \\
\text { Volcanic } \\
\text { rocks }\end{array}$ & $\begin{array}{c}\text { Percent } \\
\text { Sedimentary } \\
\text { rocks }\end{array}$ & $\begin{array}{c}\text { Percent } \\
\text { limestone }\end{array}$ & $\begin{array}{c}\text { Percent } \\
\text { coarse } \\
\text { grained } \\
\text { sedimentary } \\
\text { rocks }\end{array}$ \\
\hline 105 & 0 & 64 & 90.3 & 7.6 & 0 & 100 & 4.7 & 39.5 \\
\hline 106 & 0 & 1.5 & 268.9 & 3.9 & 0 & 100 & 1.4 & 0.5 \\
\hline 107 & 0 & 3 & 83.5 & 3.7 & 0 & 100 & 4.1 & 3.3 \\
\hline 108 & 0 & 0 & 53.3 & 0 & 0 & 100 & 0 & 0 \\
\hline 109 & 0 & 52.1 & 100.9 & 0 & 0 & 100 & 0 & 34.1 \\
\hline 110 & 0 & 100.6 & 7.9 & 0 & 0 & 100 & 0 & 92.7 \\
\hline 111 & 0 & 50.6 & 39.6 & 0 & 0 & 100 & 0 & 56.1 \\
\hline 112 & 0 & 0 & 67 & 22.9 & 0 & 100 & 25.5 & 0 \\
\hline 113 & 0 & 67.6 & 36 & 0 & 0 & 100 & 0 & 65.3 \\
\hline 114 & 0 & 91.4 & 0 & 0 & 0 & 100 & 0 & 100 \\
\hline 115 & 0 & 18.3 & 153.4 & 4.8 & 0 & 100 & 2.7 & 10.4 \\
\hline 116 & 0 & 0 & 35.8 & 13.3 & 0 & 100 & 27.1 & 0 \\
\hline 117 & 0 & 70.7 & 77.5 & 0 & 0 & 100 & 0 & 47.7 \\
\hline 118 & 0 & 0 & 31.2 & 11.4 & 0 & 100 & 26.8 & 0 \\
\hline 119 & 0 & 0 & 85.1 & 12.2 & 0 & 100 & 12.5 & 0 \\
\hline 120 & 0 & 0 & 60.6 & 30.8 & 0 & 100 & 33.7 & 0 \\
\hline 121 & 0 & 0 & 60.8 & 30.6 & 0 & 100 & 33.5 & 0 \\
\hline 122 & 0 & 41.5 & 47.1 & 2.8 & 0 & 100 & 3.1 & 45.4 \\
\hline 123 & 0 & 5.5 & 71.3 & 2.4 & 0 & 100 & 3 & 6.9 \\
\hline 124 & 1.6 & 120.4 & 231.6 & 0 & 0.5 & 99.5 & 0 & 34.2 \\
\hline 125 & 0 & 0.9 & 130.5 & 0 & 0 & 100 & 0 & 0.7 \\
\hline 126 & 0 & 0 & 116.1 & 4.3 & 0 & 100 & 3.6 & 0 \\
\hline 127 & 0 & 76.2 & 21.7 & 1.2 & 0 & 100 & 1.2 & 76.9 \\
\hline 128 & 0 & 18.6 & 98.9 & 6.9 & 0 & 100 & 5.5 & 15 \\
\hline 129 & 9.1 & 25 & 103.7 & 0 & 6.6 & 93.4 & 0 & 19.4 \\
\hline 130 & 0 & 97.5 & 0 & 0 & 0 & 100 & 0 & 100 \\
\hline 131 & 0 & 35.6 & 16.8 & 39.6 & 0 & 100 & 43 & 38.7 \\
\hline 132 & 0 & 18.3 & 91.4 & 0 & 0 & 100 & 0 & 16.7 \\
\hline 133 & 0 & 12.2 & 97.5 & 0 & 0 & 100 & 0 & 11.1 \\
\hline 134 & 0 & 76.3 & 22.8 & 0 & 0 & 100 & 0 & 77 \\
\hline 135 & 0 & 34.5 & 70.7 & 0 & 0 & 100 & 0 & 32.8 \\
\hline 136 & 0 & 0 & 40.9 & 27.7 & 0 & 100 & 40.4 & 0 \\
\hline 137 & 0 & 171.4 & 42.6 & 0 & 0 & 100 & 0 & 80.1 \\
\hline 138 & 0 & 114.3 & 0 & 0 & 0 & 100 & 0 & 100 \\
\hline 139 & 0 & 19.7 & 70.2 & 1.5 & 0 & 100 & 1.6 & 21.6 \\
\hline 140 & 0 & 82.9 & 160.9 & 0 & 0 & 100 & 0 & 34 \\
\hline 141 & 10.7 & 259.1 & 22.8 & 0 & 3.7 & 96.3 & 0 & 91.9 \\
\hline 142 & 0 & 27.5 & 93.2 & 0 & 0 & 100 & 0 & 22.8 \\
\hline 143 & 7.3 & 61.9 & 37.5 & 0 & 6.8 & 93.2 & 0 & 62.3 \\
\hline
\end{tabular}


Table 4. Lithologic data from Pahrump Valley boreholes - Continued.

[Data are compiled from the State of Nevada, Division of Water Resources, Well Driller's Reports]

\begin{tabular}{|c|c|c|c|c|c|c|c|c|}
\hline Index & $\begin{array}{l}\text { Thickness } \\
\text { of } \\
\text { volcanic } \\
\text { rock } \\
\text { (m) }\end{array}$ & $\begin{array}{c}\text { Thickness of } \\
\text { coarse grained } \\
\text { sedimentary rocks } \\
\text { (m) }\end{array}$ & $\begin{array}{l}\text { Thickness of } \\
\text { fine grained } \\
\text { sedimentary } \\
\text { rocks } \\
\text { (m) }\end{array}$ & $\begin{array}{l}\text { Limestone } \\
\text { thickness } \\
(\mathrm{m})\end{array}$ & $\begin{array}{c}\text { Percent } \\
\text { Volcanic } \\
\text { rocks }\end{array}$ & $\begin{array}{c}\text { Percent } \\
\text { Sedimentary } \\
\text { rocks }\end{array}$ & $\begin{array}{c}\text { Percent } \\
\text { limestone }\end{array}$ & $\begin{array}{c}\text { Percent } \\
\text { coarse } \\
\text { grained } \\
\text { sedimentary } \\
\text { rocks }\end{array}$ \\
\hline 144 & 0 & 151.5 & 0 & 0 & 0 & 100 & 0 & 100 \\
\hline 145 & 4.3 & 88.6 & 23.5 & 0 & 3.7 & 96.3 & 0 & 79 \\
\hline 146 & 0 & 14.1 & 89.6 & 0 & 0 & 100 & 0 & 13.6 \\
\hline 147 & 0 & 45.8 & 92.6 & 0 & 0 & 100 & 0 & 33.1 \\
\hline 148 & 0 & 39.4 & 185.3 & 2.4 & 0 & 100 & 1.1 & 17.3 \\
\hline 149 & 0 & 3.9 & 77.8 & 1.2 & 0 & 100 & 1.4 & 4.7 \\
\hline 150 & 0 & 0 & 73.7 & 14.7 & 0 & 100 & 16.6 & 0 \\
\hline 151 & 0 & 0 & 140.1 & 1.6 & 0 & 100 & 1.1 & 0 \\
\hline 152 & 0 & 157 & 0 & 0 & 0 & 100 & 0 & 100 \\
\hline 153 & 0 & 0 & 116 & 4.7 & 0 & 100 & 3.9 & 0 \\
\hline 154 & 0 & 111.3 & 0 & 0 & 0 & 100 & 0 & 100 \\
\hline 155 & 0 & 103.6 & 0 & 0 & 0 & 100 & 0 & 100 \\
\hline 156 & 0 & 15.9 & 68.8 & 0 & 0 & 100 & 0 & 18.8 \\
\hline 157 & 0 & 74.7 & 13.7 & 51.9 & 0 & 100 & 37 & 53.2 \\
\hline 158 & 0 & 131.2 & 107.5 & 0 & 0 & 100 & 0 & 55 \\
\hline 159 & 0 & 152.4 & 0 & 0 & 0 & 100 & 0 & 100 \\
\hline 160 & 0 & 22.3 & 121.2 & 11.9 & 0 & 100 & 7.7 & 14.4 \\
\hline 161 & 0 & 4.6 & 129.3 & 18.5 & 0 & 100 & 12.1 & 3 \\
\hline 162 & 0 & 54.8 & 48.8 & 0 & 0 & 100 & 0 & 52.9 \\
\hline 163 & 0 & 54.2 & 41.8 & 0 & 0 & 100 & 0 & 56.5 \\
\hline 164 & 45.7 & 6 & 51.9 & 16.8 & 38 & 62 & 14 & 8 \\
\hline 165 & 0 & 0 & 157 & 0 & 0 & 100 & 0 & 0 \\
\hline 166 & 0 & 76.2 & 157 & 0 & 0 & 100 & 0 & 32.7 \\
\hline 167 & 0 & 146.3 & 160 & 0 & 0 & 100 & 0 & 47.8 \\
\hline 168 & 0 & 26.8 & 76.2 & 0 & 0 & 100 & 0 & 26 \\
\hline 169 & 0 & 18.6 & 80.1 & 0.6 & 0 & 100 & 0.6 & 18.7 \\
\hline 170 & 0 & 0 & 91.4 & 0 & 0 & 100 & 0 & 0 \\
\hline 171 & 0 & 5.4 & 82.3 & 0 & 0 & 100 & 0 & 6.2 \\
\hline 172 & 0 & 32.3 & 34.7 & 0 & 0 & 100 & 0 & 48.2 \\
\hline 173 & 0 & 11.6 & 103.6 & 0 & 0 & 100 & 0 & 10.1 \\
\hline 174 & 0 & 115.8 & 0 & 0 & 0 & 100 & 0 & 100 \\
\hline 175 & 0 & 115.2 & 0 & 0 & 0 & 100 & 0 & 100 \\
\hline 176 & 0 & 0 & 93.9 & 2.4 & 0 & 100 & 2.5 & 0 \\
\hline 177 & 0 & 3.5 & 112.4 & 5.4 & 0 & 100 & 4.5 & 2.9 \\
\hline 178 & 0 & 62.2 & 48.7 & 0 & 0 & 100 & 0 & 56.1 \\
\hline 179 & 0 & 109.7 & 44.2 & 0 & 0 & 100 & 0 & 71.3 \\
\hline 180 & 0 & 111.9 & 61 & 3 & 0 & 100 & 1.7 & 63.6 \\
\hline 181 & 0 & 0 & 80.2 & 11.2 & 0 & 100 & 12.3 & 0 \\
\hline 182 & 0 & 3.9 & 82 & 5.5 & 0 & 100 & 6 & 4.3 \\
\hline
\end{tabular}


Table 4. Lithologic data from Pahrump Valley boreholes - Continued.

[Data are compiled from the State of Nevada, Division of Water Resources, Well Driller's Reports]

\begin{tabular}{|c|c|c|c|c|c|c|c|c|}
\hline Index & $\begin{array}{l}\text { Thickness } \\
\text { of } \\
\text { volcanic } \\
\text { rock } \\
\text { (m) }\end{array}$ & $\begin{array}{c}\text { Thickness of } \\
\text { coarse grained } \\
\text { sedimentary rocks } \\
(\mathrm{m})\end{array}$ & $\begin{array}{l}\text { Thickness of } \\
\text { fine grained } \\
\text { sedimentary } \\
\text { rocks } \\
\text { (m) }\end{array}$ & $\begin{array}{c}\text { Limestone } \\
\text { thickness } \\
\text { (m) }\end{array}$ & $\begin{array}{l}\text { Percent } \\
\text { Volcanic } \\
\text { rocks }\end{array}$ & $\begin{array}{c}\text { Percent } \\
\text { Sedimentary } \\
\text { rocks }\end{array}$ & $\begin{array}{c}\text { Percent } \\
\text { limestone }\end{array}$ & $\begin{array}{c}\text { Percent } \\
\text { coarse } \\
\text { grained } \\
\text { sedimentary } \\
\text { rocks }\end{array}$ \\
\hline 183 & 0 & 0 & 77.4 & 44.5 & 0 & 100 & 36.5 & 0 \\
\hline 184 & 0 & 19.8 & 71.6 & 0 & 0 & 100 & 0 & 21.7 \\
\hline 185 & 0 & 66.1 & 115.9 & 0 & 0 & 100 & 0 & 36.3 \\
\hline 186 & 0 & 121.9 & 114.3 & 12.2 & 0 & 100 & 4.9 & 49.1 \\
\hline 187 & 0 & 0 & 91.4 & 0 & 0 & 100 & 0 & 0 \\
\hline 188 & 0 & 0 & 91.4 & 0 & 0 & 100 & 0 & 0 \\
\hline 189 & 0 & 0 & 306.3 & 0 & 0 & 100 & 0 & 0 \\
\hline 190 & 0 & 0 & 144.8 & 0 & 0 & 100 & 0 & 0 \\
\hline 191 & 0 & 0 & 310.9 & 0 & 0 & 100 & 0 & 0 \\
\hline 192 & 0 & 0 & 121.9 & 0 & 0 & 100 & 0 & 0 \\
\hline 193 & 0 & 0 & 99.4 & 0 & 0 & 100 & 0 & 0 \\
\hline 194 & 0 & 0 & 108.2 & 0 & 0 & 100 & 0 & 0 \\
\hline 195 & 0 & 0 & 210.3 & 0 & 0 & 100 & 0 & 0 \\
\hline 196 & 0 & 0 & 201.8 & 0 & 0 & 100 & 0 & 0 \\
\hline 197 & 0 & 0 & 182.9 & 0 & 0 & 100 & 0 & 0 \\
\hline 198 & 0 & 0 & 121.9 & 0 & 0 & 100 & 0 & 0 \\
\hline 199 & 0 & 0 & 91.4 & 0 & 0 & 100 & 0 & 0 \\
\hline 200 & 0 & 0 & 100.6 & 0 & 0 & 100 & 0 & 0 \\
\hline 201 & 0 & 0 & 91.4 & 0 & 0 & 100 & 0 & 0 \\
\hline 202 & 0 & 0 & 114.3 & 0 & 0 & 100 & 0 & 0 \\
\hline 203 & 0 & 0 & 114.3 & 0 & 0 & 100 & 0 & 0 \\
\hline 204 & 0 & 0 & 96.6 & 0 & 0 & 100 & 0 & 0 \\
\hline 205 & 0 & 0 & 109.7 & 0 & 0 & 100 & 0 & 0 \\
\hline 206 & 0 & 0 & 100.6 & 0 & 0 & 100 & 0 & 0 \\
\hline 207 & 0 & 0 & 129.5 & 0 & 0 & 100 & 0 & 0 \\
\hline 208 & 0 & 0 & 135.6 & 0 & 0 & 100 & 0 & 0 \\
\hline 209 & 0 & 0 & 139 & 0 & 0 & 100 & 0 & 0 \\
\hline 210 & 0 & 0 & 137.2 & 0 & 0 & 100 & 0 & 0 \\
\hline 211 & 0 & 0 & 236.2 & 0 & 0 & 100 & 0 & 0 \\
\hline 212 & 0 & 0 & 152.4 & 0 & 0 & 100 & 0 & 0 \\
\hline 213 & 0 & 0 & 106.7 & 0 & 0 & 100 & 0 & 0 \\
\hline 214 & 0 & 0 & 114.3 & 0 & 0 & 100 & 0 & 0 \\
\hline 215 & 0 & 0 & 120.4 & 0 & 0 & 100 & 0 & 0 \\
\hline 216 & 0 & 0 & 121.9 & 0 & 0 & 100 & 0 & 0 \\
\hline 217 & 0 & 0 & 182.9 & 0 & 0 & 100 & 0 & 0 \\
\hline 218 & 0 & 0 & 121.9 & 0 & 0 & 100 & 0 & 0 \\
\hline 219 & 0 & 0 & 167.6 & 0 & 0 & 100 & 0 & 0 \\
\hline 220 & 0 & 0 & 138.7 & 0 & 0 & 100 & 0 & 0 \\
\hline 221 & 0 & 0 & 105.2 & 0 & 0 & 100 & 0 & 0 \\
\hline 222 & 0 & 0 & 149.4 & 0 & 0 & 100 & 0 & 0 \\
\hline
\end{tabular}


Table 4. Lithologic data from Pahrump Valley boreholes - Continued.

[Data are compiled from the State of Nevada, Division of Water Resources, Well Driller's Reports]

\begin{tabular}{|c|c|c|c|c|c|c|c|c|}
\hline Index & $\begin{array}{l}\text { Thickness } \\
\text { of volcanic } \\
\text { rock } \\
\text { (m) }\end{array}$ & $\begin{array}{c}\text { Thickness of } \\
\text { coarse grained } \\
\text { sedimentary rocks } \\
(\mathrm{m})\end{array}$ & $\begin{array}{l}\text { Thickness of } \\
\text { fine grained } \\
\text { sedimentary } \\
\text { rocks } \\
\text { (m) }\end{array}$ & $\begin{array}{l}\text { Limestone } \\
\text { thickness } \\
\text { (m) }\end{array}$ & $\begin{array}{c}\text { Percent } \\
\text { Volcanic } \\
\text { rocks }\end{array}$ & $\begin{array}{c}\text { Percent } \\
\text { Sedimentary } \\
\text { rocks }\end{array}$ & $\begin{array}{c}\text { Percent } \\
\text { limestone }\end{array}$ & $\begin{array}{c}\text { Percent } \\
\text { coarse } \\
\text { grained } \\
\text { sedimentary } \\
\text { rocks } \\
\end{array}$ \\
\hline 223 & 0 & 0 & 242.3 & 0 & 0 & 100 & 0 & 0 \\
\hline 224 & 0 & 0 & 246.3 & 0 & 0 & 100 & 0 & 0 \\
\hline 225 & 0 & 0 & 128 & 0 & 0 & 100 & 0 & 0 \\
\hline 226 & 0 & 0 & 128.3 & 0 & 0 & 100 & 0 & 0 \\
\hline 227 & 0 & 0 & 121.9 & 0 & 0 & 100 & 0 & 0 \\
\hline 228 & 0 & 0 & 128 & 0 & 0 & 100 & 0 & 0 \\
\hline 229 & 0 & 0 & 97.5 & 0 & 0 & 100 & 0 & 0 \\
\hline 230 & 0 & 0 & 167.6 & 0 & 0 & 100 & 0 & 0 \\
\hline 231 & 0 & 0 & 259.4 & 0 & 0 & 100 & 0 & 0 \\
\hline 232 & 0 & 0 & 121.9 & 0 & 0 & 100 & 0 & 0 \\
\hline 233 & 0 & 0 & 121.9 & 0 & 0 & 100 & 0 & 0 \\
\hline 234 & 0 & 0 & 121.9 & 0 & 0 & 100 & 0 & 0 \\
\hline 235 & 0 & 0 & 134.1 & 0 & 0 & 100 & 0 & 0 \\
\hline 236 & 0 & 0 & 121.9 & 0 & 0 & 100 & 0 & 0 \\
\hline 237 & 0 & 0 & 290.2 & 0 & 0 & 100 & 0 & 0 \\
\hline 238 & 0 & 0 & 121.9 & 0 & 0 & 100 & 0 & 0 \\
\hline 239 & 0 & 0 & 105.2 & 0 & 0 & 100 & 0 & 0 \\
\hline 240 & 0 & 0 & 152.4 & 0 & 0 & 100 & 0 & 0 \\
\hline 241 & 0 & 0 & 128.6 & 0 & 0 & 100 & 0 & 0 \\
\hline 242 & 0 & 0 & 121.9 & 0 & 0 & 100 & 0 & 0 \\
\hline 243 & 0 & 0 & 121.9 & 0 & 0 & 100 & 0 & 0 \\
\hline 244 & 0 & 0 & 152.4 & 0 & 0 & 100 & 0 & 0 \\
\hline 245 & 0 & 0 & 92.4 & 0 & 0 & 100 & 0 & 0 \\
\hline 246 & 0 & 0 & 147.8 & 0 & 0 & 100 & 0 & 0 \\
\hline 247 & 0 & 0 & 160 & 0 & 0 & 100 & 0 & 0 \\
\hline 248 & 0 & 0 & 98.1 & 0 & 0 & 100 & 0 & 0 \\
\hline 249 & 0 & 0 & 93 & 0 & 0 & 100 & 0 & 0 \\
\hline 250 & 0 & 0 & 121.9 & 0 & 0 & 100 & 0 & 0 \\
\hline 251 & 0 & 0 & 152.4 & 0 & 0 & 100 & 0 & 0 \\
\hline 252 & 0 & 0 & 104.2 & 0 & 0 & 100 & 0 & 0 \\
\hline 253 & 0 & 60.7 & 60 & 0 & 0 & 100 & 0 & 50.3 \\
\hline 254 & 0 & 46.6 & 47.2 & 3.4 & 0 & 100 & 3.4 & 48 \\
\hline 255 & 0 & 0 & 86.3 & 11.3 & 0 & 100 & 11.6 & 0 \\
\hline
\end{tabular}


Table 4. Lithologic data from Pahrump Valley boreholes - Continued.

[Data are compiled from the State of Nevada, Division of Water Resources, Well Driller's Reports]

\begin{tabular}{ccccccccc}
\hline Index & $\begin{array}{c}\text { Thickness } \\
\text { of } \\
\text { volcanic } \\
\text { rock } \\
(\mathrm{m})\end{array}$ & $\begin{array}{c}\text { Thickness of } \\
\text { coarse grained } \\
\text { sedimentary rocks } \\
(\mathrm{m})\end{array}$ & $\begin{array}{c}\text { Thickness of } \\
\text { fine grained } \\
\text { sedimentary } \\
\text { rocks } \\
(\mathrm{m})\end{array}$ & $\begin{array}{c}\text { Limestone } \\
\text { thickness } \\
(\mathrm{m})\end{array}$ & $\begin{array}{c}\text { Percent } \\
\text { Volcanic } \\
\text { rocks }\end{array}$ & $\begin{array}{c}\text { Percent } \\
\text { Sedimentary } \\
\text { rocks }\end{array}$ & $\begin{array}{c}\text { Percent } \\
\text { limestone }\end{array}$ & $\begin{array}{c}\text { Percent } \\
\text { coarse } \\
\text { grained } \\
\text { sedimentary } \\
\text { rocks }\end{array}$ \\
\hline 256 & 0 & 22.3 & 50.9 & 0.9 & 0 & 100 & 1.2 & 30 \\
257 & 0 & 82.3 & 36.3 & 3.4 & 0 & 100 & 2.8 & 67.5 \\
258 & 0 & 0 & 130.5 & 6.7 & 0 & 100 & 4.9 & 0 \\
259 & 0 & 1.2 & 55.5 & 4 & 0 & 100 & 6.5 & 2 \\
260 & 0 & 0 & 52.1 & 1.5 & 0 & 100 & 2.8 & 0 \\
261 & 0 & 0 & 17.7 & 40.2 & 0 & 100 & 69.5 & 0 \\
262 & 0 & 0 & 142.9 & 3.4 & 0 & 100 & 2.3 & 0 \\
263 & 0 & 36.9 & 167.3 & 0 & 0 & 100 & 0 & 18.1 \\
264 & 0 & 12.5 & 139.9 & 0 & 0 & 100 & 0 & 8.2 \\
265 & 0 & 126.2 & 118.9 & 16.8 & 0 & 100 & 6.4 & 48.2 \\
266 & 10.7 & 228.6 & 50.3 & 0 & 3.7 & 96.3 & 0 & 82 \\
267 & 0 & 12.25 & 0 & 0 & 0 & 100 & 0 & 100 \\
268 & 0 & 24 & 0 & 0 & 0 & 100 & 0 & 100 \\
269 & 0 & 39 & 0 & 0 & 0 & 100 & 0 & 100 \\
270 & 0 & 35 & 0 & 0 & 0 & 100 & 0 & 100 \\
\hline
\end{tabular}




\section{Compositional Trends}

The basin-filling rocks of the Pahrump basin are almost entirely composed of sedimentary rocks. Only two boreholes, out of 266, were initially interpreted as containing more than a few percent volcanic rocks, based on driller's descriptions: well PB11478 and well PB61022 (table 2). Well PB11478 reportedly intercepted about $16 \mathrm{~m}$ of "fractured, black rock" between 172 and $188 \mathrm{~m}$ depth; this was interpreted to correspond to basalt. This well is located along the southern edge of the Pahrump fan, 3 $\mathrm{km}$ from outcrops of Paleozoic limestone. Based on proximity to bedrock outcrops and an absence of any detectable aeromagnetic anomaly (Blakely and others, 2000), it is likely that this borehole intercepted dark-colored Paleozoic limestone bedrock at depth. Well PB61022 reportedly intercepted $46 \mathrm{~m}$ of "hard granite" between 10 and $56 \mathrm{~m}$ depth. This well is located at the north end of the Pahrump Valley; no tuffs are known to crop out in the vicinity. A possible interpretation is that the "hard granite" represents coarse colluvial material derived from the Late Proterozoic Johnnie Formation and Stirling Quartzite that crop out on the northwestern flank of the Spring Mountains.

The basin filling sediments are divided between coarse-grained sedimentary rocks of the Manse and Pahrump fans on the eastern and northeastern flank of the basin, and finegrained siliciclastic rocks and limestone along the basin axis to the southwest (fig. 5). Borehole lithologic data from the coarse-grained fan are nearly lacking; only the fan toe is well represented by borehole data. Lithologic data were contoured (fig. 5) using a hybrid routine that combines triangulation and inverse distance methods, giving equal weight to both routines. The boundary between coarse-grained and fine-grained rocks mimics the shape and location of the currently mapped toe of the fan (Malmberg, 1967; Lundstrom and others, in press; Page and others, in press). In detail, the transition from alluvial fan to basin-axis deposits is characterized by complex interfingering of coarsegrained and fine-grained rocks (fig. 3). Within the fine-grained sediments along the basin axis, individual beds of limestone are not readily correlated, even over short distances (fig. 3).

\section{Summary}

Driller's logs from water wells in the Pahrump Valley, NV-CA provide a readily available source of subsurface information. Driller's descriptions, when converted to a uniform suite of lithologic descriptors, form a reasonably self-consistent set of data that depict the spatial variaibility of rock types within the upper basin fill. Compilation and interpretation of these data represent the initial step in developing a three-dimensional understanding of the geometry and properties of the subsurface materials within this basin. 


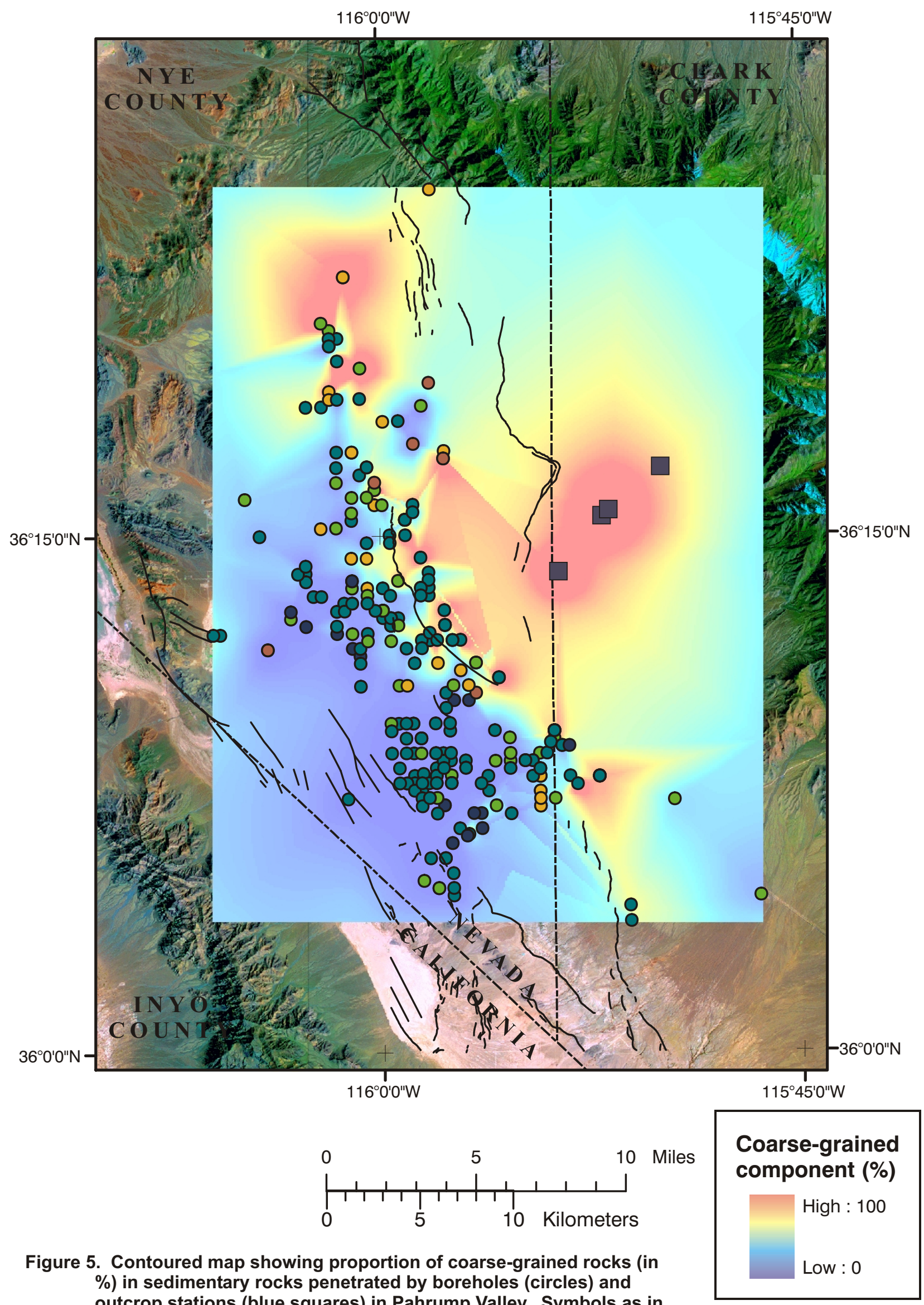

$\%$ ) in sedimentary rocks penetrated by boreholes (circles) and outcrop stations (blue squares) in Pahrump Valley. Symbols as in figure 2. 


\section{References Cited}

Anderson, R.E., Bucknam, R.C., Crone, A.J., Haller, K.M., Machette, M.N., Personius, S.F., Barnard, T.P., Cecil, M.J., and Dart, R.L., 1995, Characterization of Quaternary and suspected Quaternary faults, regional studies, Nevada and California: U.S. Geological Survey Open-File Report 95-599, 56 p.

Blakely, R.J., Jachens, R.C., Calzia, J.P., and Langenheim, V.E., 1999, Cenozoic basins of the Death Valley extended terrane as reflected in regional gravity anomalies, in Wright. L.A., and Troxel, B.W., eds., Cenozoic basins of the Death Valley Region: Geological Society of America Special Paper 333, p. 1-16.

Blakely, R.J., Langenheim, V.E., Ponce, D.A., and Dixon, G.L., 2000, Aeromagnetic survey of the Amargosa Desert, Nevada and California: A tool for understanding near-surface geology and hydrology: U.S. Geological Survey Open-File Report 00188,39 p., 2 plates.

Blakely, R.J., Morin, R.L., McKee, E.H., Schmidt, K.M., Langenheim, V.E., and Dixon, G.L., 1998, Three-dimensional model of pre-Cenozoic basement beneath Amargosa Desert and Pahrump Valley, California and Nevada - Implications for tectonic evolution and water resources: U.S. Geological Survey Open-File-Report 98-496, 29 p.

Burchfiel, B.C., Fleck, R.J., Secor, D.T., Vincelette, R.R., and Davis, G.A., 1974, Geology of the Spring Mountains: Geological Society of America Bulletin, v. 85, p. 1013-1022.

Burchfiel, B.C., Hamill, G.S., and Wilhelms, D.E., 1982, Stratigraphy of the Montgomery Mountains and the northern half of the Nopah and Resting Springs Ranges, Nevada and California: Geological Society of America Map and Chart Series MC-44, scale 1:62,5000.

1983, Structural geology of the Montgomery Mountains and the northern half of the Nopah and Resting Springs Ranges, Nevada and California: Geological Society of America Bulletin, v. 94, p. 1359-1376.

Calzia, J.P., Troxel, B.W., Wright, L.A., Burchfiel, B.C., Davis, G.A., and McMackin, M.R., 2000, Geologic map of the Kingston Range, southern Death Valley, California: U.S. Geological Survey Open-File-Report 00-412, scale 1:31,680, 2 plates.

dePolo, C.M., Ramelli, A.R., and Bell, J.W., 1999, Geologic map of the Pahrump Quadrangle, Nevada: Nevada Bureau of Mines and Geology, Open-File Report 99-14, 1:24,000 scale.

Harrill, J.R., 1986, Ground-water storage depletion in Pahrump Valley, NevadaCalifornia, 1962-75: U.S. Geological Survey Water-Supply Paper 2279, 52 p., 3 plates. 
Hoffard, J.L., 1991, Quaternary tectonics and basin history of Pahrump and Stewart Valleys; Unpublished M.S. thesis, University of Nevada, Reno, Nevada.

Longwell, C.R., Pampeyan, E.H., Bowyer, Ben, and Roberts, R.J., 1965, Geology and mineral deposits of Clark County, Nevada: Nevada Bureau of Mines and Geology Bulletin 62, $218 \mathrm{p}$.

Lundstrom, S.C, Mahan, S.A., Blakely, R.J., Paces, J.B., Dixon, G.L., and Young, O.D., in press, Geologic map of the Mound Spring quadarangle, Nye and Clark Counties, Nevada, and Inyo County, California: U.S. Geological Survey Miscellaneous Field Studies Map MF-2339, scale 1:24,000.

Malmberg, G.T., 1967, Hydrology of the valley-fill and carbonate-rock reservoirs, Pahrump Valley, Nevada-California: U.S. Geological Survey Water Supply Paper 1832, 46 p., 5 plates.

Page, W.R., Lundstrom, S.C., Harris, A.G., Workman, J.B., Mahan, S.A., Paces, J.B., Morgan, K.S., Kempton, E.C., Rowley, P.D., Langenheim, V.E., Dixon, G.L., Young, O.D., Burchfiel, B.C., Bell, J.W., and Smith, E.I., in press, Geologic Map of the Las Vegas 30 x 60-minute quadrangle, Clark and Nye Counties, Nevada, and Inyo County, California: U.S. Geological Survey Miscellaneous Investigations Map, scale 1:100,000.

Piety, L. A., 1996, Compilation of known or suspected Quaternary faults within $100 \mathrm{~km}$ of Yucca Mountain , Nevada and California: U.S. Geological Survey Open-File Report 94-112, 404 p., 2 plates.

Stewart, J.H., Albers, J.P., and Poole, F.G., 1968, Summary of regional evidence for right-lateral displacement in the western Great Basin: Geological Society of America Bulletin, v. 79, p. 1407-1413.

Stewart, J.H., and Crowell, J.C., 1992, Strike-slip tectonics in the Cordillera region, western United States, in Burchfiel, B.C., Lipman, P.W., and Zoback, M.L., eds., The Cordilleran orogen in conterminous U.S.: Geological Society of America, Geology of North America, v. H-3, p. 609-628.

Workman, J.B., Menges, C.M., Page, W.R., Taylor, E.M., Ekren, E.B., Rowley, P.D., Dixon, G.L., Thompson, R.A., and Wright, L.A., 2002, Geologic map of the Death Valley ground-water model area, Nevada and California: U.S. Geological Survey Miscellaneous Field Studies Map MF-2381-A, scale 1:250,000.

Wright, L.A., 1989, Overview of the role of strike-slip and normal faulting in the Neogene history of the region northeast of Death Valley, California-Nevada, in Ellis,M.A., ed., Late Cenozoic evolution of the southern Great Basin: Nevada Bureau of Mines and Geology Open-File Report 89-1, p. 1-11. 